\title{
Brain indoleamine 2,3-dioxygenase contributes to the comorbidity of pain and depression
}

\author{
Hyangin Kim, Lucy Chen, Grewo Lim, Backil Sung, Shuxing Wang, Michael F. McCabe, \\ Gabriel Rusanescu, Liling Yang, Yinghong Tian, and Jianren Mao \\ MGH Center for Translational Pain Research, Department of Anesthesia, Critical Care and Pain Medicine, Massachusetts General Hospital, \\ Harvard Medical School, Boston, Massachusetts, USA.
}

\begin{abstract}
Pain and depression are frequently comorbid disorders, but the mechanism underlying this association is unknown. Here, we report that brain indoleamine 2,3-dioxygenase 1 (IDO1), a rate-limiting enzyme in tryptophan metabolism, plays a key role in this comorbidity. We found that chronic pain in rats induced depressive behavior and IDO1 upregulation in the bilateral hippocampus. Upregulation of IDO1 resulted in the increased kynurenine/tryptophan ratio and decreased serotonin/tryptophan ratio in the bilateral hippocampus. We observed elevated plasma IDO activity in patients with both pain and depression, as well as in rats with anhedonia induced by chronic social stress. Intra-hippocampal administration of IL-6 in rats, in addition to in vitro experiments, demonstrated that IL-6 induces IDO1 expression through the JAK/STAT pathway. Further, either Ido1 gene knockout or pharmacological inhibition of hippocampal IDO1 activity attenuated both nociceptive and depressive behavior. These results reveal an IDO1-mediated regulatory mechanism underlying the comorbidity of pain and depression and suggest a new strategy for the concurrent treatment of both conditions via modulation of brain IDO1 activity.
\end{abstract}

\section{Introduction}

Pain and depression often coexist in the clinical setting, which complicates the treatment of both conditions. The prevalence rate of depression is several times higher in patients with chronic pain than in the general population (1), whereas depression significantly increases the risk of developing chronic pain (2). Currently, antidepressants and analgesics are often prescribed in combination for symptomatic management, but this clinical approach has achieved only limited success (3). To date, the cellular mechanism underlying the comorbid relationship between pain and depression remains unclear.

Tryptophan is an essential amino acid and the precursor of serotonin and kynurenine, two neuromodulators critically implicated in the regulation of neuronal excitation (4) and depression (5). Indoleamine 2,3-dioxygenase 1 (IDO1) is a rate-limiting enzyme in tryptophan metabolism. Relative to its basal expression in immune cells, IDO1 is significantly upregulated in response to inflammation $(5,6)$. Recent studies in the depression and immunology fields have shown that IDO1 activity is linked to (a) decreased serotonin content (1) and depression (1, 5) and (b) increased kynurenine content and neuroplastic changes through the effect of its derivatives such as quinolinic acid on glutamate receptors (7). Moreover, IDO1 expression has been shown to be induced by proinflammatory cytokines, leading to the increased kynurenine production (8-10).

Since proinflammatory cytokines including IL- 6 have been implicated in the pathophysiology of both pain (11) and depression (12), it is possible that regulation of brain IDO1 by proinflammatory cytokines could serve as a critical mechanistic link in the comorbid relationship between pain and depression through

Conflict of interest: The authors have declared that no conflict of interest exists. Citation for this article: J Clin Invest. 2012;122(8):2940-2954. doi:10.1172/JCI61884. the regulation of tryptophan metabolism. We tested this hypothesis by utilizing a rat model of induced depressive behavior resulting from persistent hind paw inflammatory pain (13).

\section{Results}

Persistent nociception induces depressive behavior. Inflammatory arthritis in Wistar rats induced by the injection of CFA into the right tibiotarsal joint produced mechanical allodynia (Figure 1A; ANOVA, $F(3,104)=3.11, P<0.05)$ and thermal hyperalgesia (Figure $1 \mathrm{~B} ; F(3,121)=8.99, P<0.05)$, which lasted for at least 21 days as compared with sham control rats injected with incomplete Freund's adjuvant. This condition of persistent nociception induced depressive behavior in these same rats when examined on days 7 and 14, but not on day 1 , in the forced swimming test (FST) (ref. 14 and Figure $1 C ; F(3,37)=18.91 ; P<0.01)$ and open field test (OFT) (refs. 15, 16, and Figure 1D; $F(3,31)=6.08, P<0.05$ ). A shorter hind paw withdrawal latency in arthritic rats correlated with a longer immobility time in FST (Figure 1E) and a lower frequency in OFT (Figure 1F), demonstrating a comorbid relationship between pain and depression in these rats. Of note, the increased immobility time in FST and reduced frequency in OFT were observed in both arthritic and sham control rats on day 1 but only in arthritic rats on day 7 and day 14. Testing of depressive behavior was not extended beyond day 14 in order to avoid habituation to the testing environment, because there were no differences after day 14 in nociceptive behavior. The intensity of exploratory behavior (e.g., rearing and crossing in OFT) was similar in arthritic and sham control rats, although arthritic rats had a lower frequency of exploratory behaviors. Moreover, there were no differences in a rotarod test between rats with or without hind paw arthritis on day 7 (Supplemental Figure 1A; supplemental material available online with this article; doi:10.1172/JCI61884DS1), suggesting that the observed depressive behavior was unlikely due to changes in motor function. 

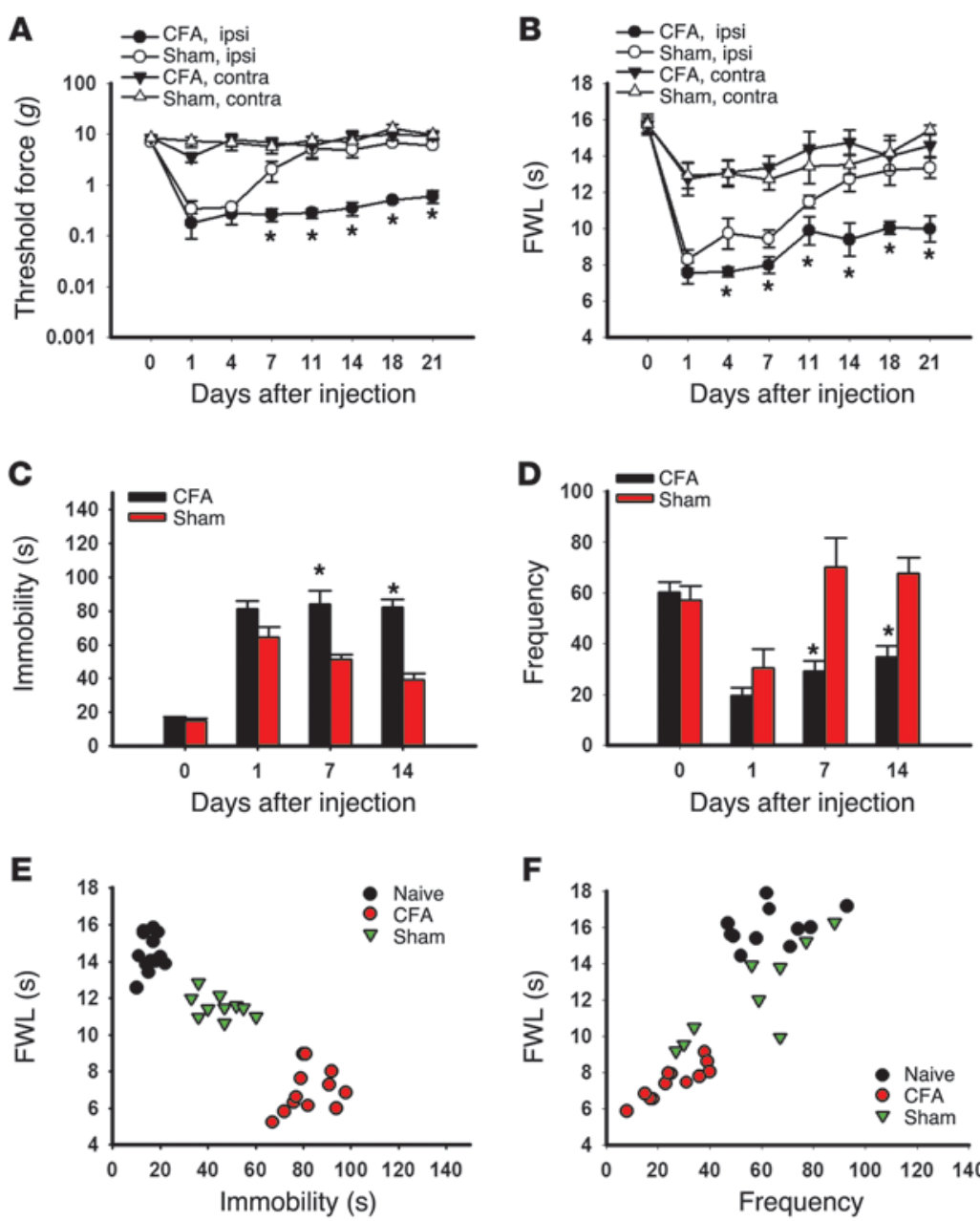
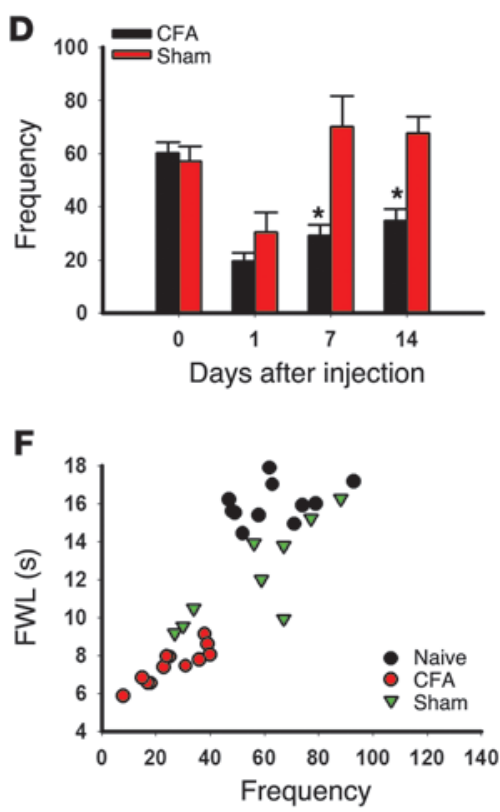

\section{Figure 1}

Correlation of nociceptive and depressive behaviors. (A-D) The injection of CFA into the right tibiotarsal joint of Wistar rats produced mechanical allodynia $(\mathbf{A})$ and thermal hyperalgesia (B) on the ipsilateral hind paw, as well as prolonging the immobility time in FST (C) and reducing the frequency (number of squares crossed) in OFT (D). Mean \pm SEM, $n=10-11$, ${ }^{*} P<0.05$, compared with sham controls. FWL, foot withdrawal latency; ipsi, ipsilateral; contra, contralateral. (E) The prolonged immobility time in FST was inversely correlated with the reduced hind paw withdrawal latency in the thermal hyperalgesia test. The data were obtained on day 14 . $r=-0.913, n=10-11,{ }^{*} P<0.01$. (F) The reduced frequency in OFT correlated with the reduced hind paw withdrawal latency in the thermal hyperalgesia test. $r=0.840, n=10-11, P<0.01$.
Hippocampal IDO1 expression is upregulated in rats with coexistent nociceptive and depressive behavior. We first examined whether brain IDO1 expression (hippocampus, thalamus, and nucleus accumbens) would differ in rats with or without coexistent nociceptive and depressive behavior. IDO1 immunoreactivity in the hippocampus (Figure 2A) was co-localized with glial fibrillary acidic protein (GFAP; astrocyte marker), Iba-1 (microglial marker), and NeuN (neuronal marker) (Figure 2B), consistent with both in vivo and in vitro expression of IDO1 in immune cells and neurons $(17,18)$. While the basal Ido1 mRNA level (real-time PCR) in the bilateral hippocampus was similar in arthritic and sham rats (naive, day 0; contralateral side only), the Ido1 mRNA level was progressively increased on days 1,7 , and 14 in arthritic but not sham rats (Figure 2C; $P<0.05$ ). The IDO1 protein level (Western blot analysis) was also elevated in the hippocampus (Figure $2 \mathrm{D}$; contralateral side only, $P<0.05)$, but not in the thalamus or nucleus accumbens (Figure 2, E and F), of arthritic rats. Moreover, there was a temporal relationship between IDO1 upregulation and nociceptive (Figure 1, A and B) and depressive behavior (Figure $1, \mathrm{C}$ and $\mathrm{E}$ ) in these same rats.

Increased IDO1 enzyme activity alters ratios of hippocampal tryptophan metabolites. To examine the role of IDO1 enzyme activity in tryptophan metabolism in both arthritic and sham rats, we first measured the content of tryptophan, serotonin, and kynurenine in the hippocampus using HPLC and then deter- mined the ratio of serotonin or kynurenine to tryptophan. There were no baseline differences in the kynurenine/tryptophan or serotonin/tryptophan ratio between arthritic and sham control rats (Figure 3, A and B). However, the kynurenine/tryptophan ratio was significantly increased (Figure 3A; $P<0.05$ ), while the serotonin/tryptophan ratio was decreased (Figure 3B; $P<0.05$ ), in arthritic rats as compared with sham control rats when measured on both day 1 and day 14. In contrast, IDO1 enzyme activity (both kynurenine/tryptophan and serotonin/tryptophan ratios) was not changed in the thalamus of arthritic rats (Figure 3, C and D). In addition, the plasma kynurenine/tryptophan ratio was also significantly increased (Figure 3E; $P<0.05$ ) in arthritic rats as compared with sham control rats, although the plasma serotonin/tryptophan ratio remained unchanged (Figure 3F), when both were examined on day 14. Consistent with the IDO1 upregulation in arthritic rats, altered ratios of tryptophan metabolites in the hippocampus indicate increased IDO1 enzyme activity in rats with coexistent nociceptive and depressive behavior.

Patients with both chronic back pain and depression also showed a significantly elevated plasma IDO1 level (Figure 3G) and increased IDO1 enzyme activity (Figure $3 \mathrm{H}$; increased kynurenine/tryptophan ratio) as compared with healthy control subjects without pain and depression $(P<0.05)$. In contrast, the serotonin/tryptophan ratio was not different between 
A
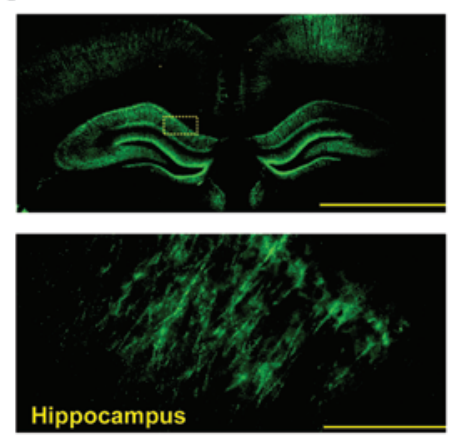

C
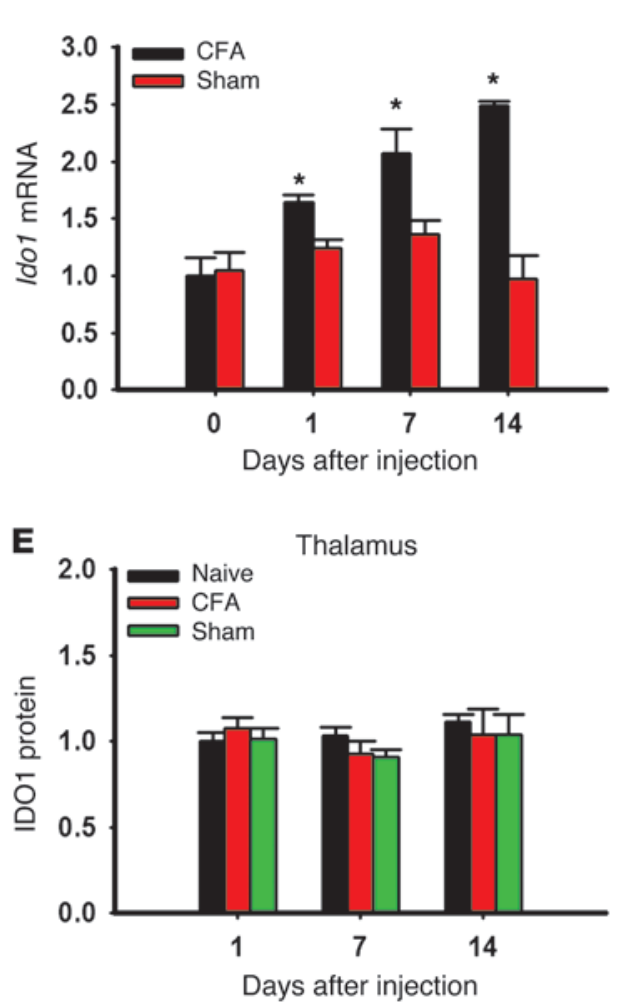

Days after injection
B

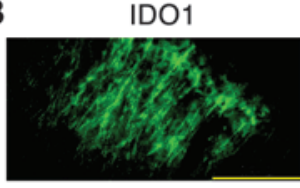

IDO1
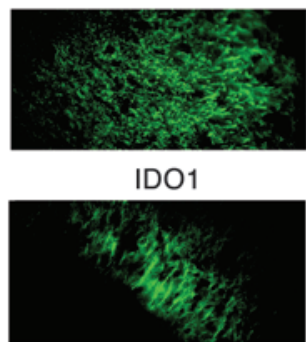

D

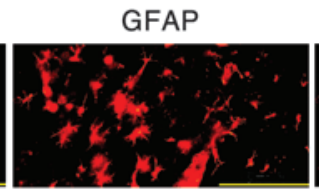

Iba-1

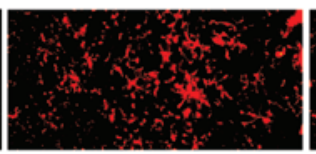

NeuN

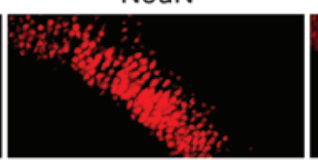

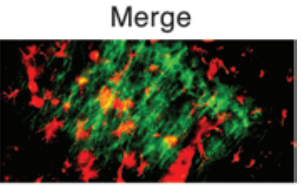

Merge

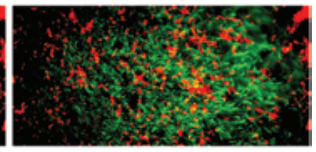

Merge

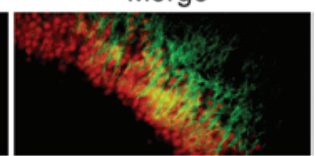

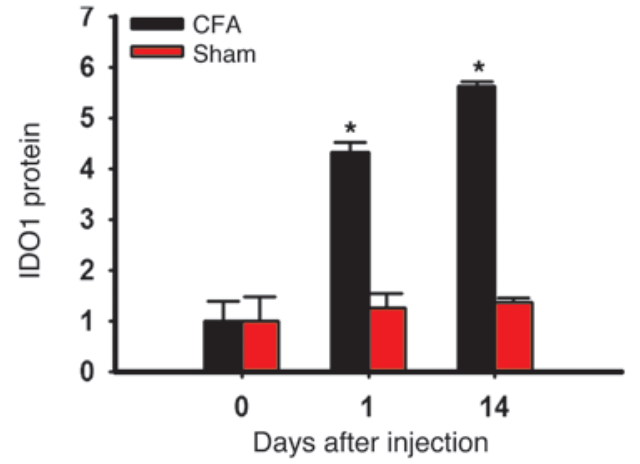

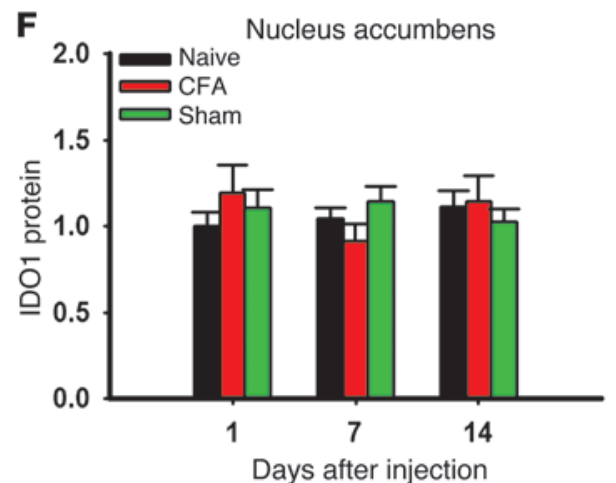

\section{Figure 2}

IDO1 expression in the hippocampus. (A) IDO1 immunoreactivity was detected in the hippocampus. Scale bars: $1.0 \mathrm{~mm}$ (top), $50 \mu \mathrm{m}$ (bottom). (B) Photomicrographs of colocalization between IDO1 and GFAP, Iba-1, or NeuN in the hippocampus. Scale bars: $50 \mu \mathrm{m}$. (C and D) Ido1 mRNA (C) and protein (D) expression was increased in the contralateral hippocampus of Wistar rats injected with CFA as detected by real-time PCR (C) and Western blot analysis (D). Day 0, baseline (naive rats); C-1 and C-14, samples taken on day 1 and day 14 from rats with CFA-induced arthritis; S-1 and S-14, samples taken on day 1 and day 14 from sham control rats. $\beta$-Actin was used as loading control. $y$ axis shows fold change in IDO1 mRNA and protein expression. Mean \pm SEM, $n=6-10,{ }^{*} P<0.05$ compared with sham control. (E and F) IDO1 expression (Western blot) was not upregulated in the thalamus $(E)$ or nucleus accumbens $(\mathbf{F})$ in Wistar rats with CFA-induced arthritis; $n=6, P>0.05$.

healthy control subjects and patients with both pain and depression (Figure 3I). Although the data were obtained in a cross-sectional observational setting, these findings suggest that a relationship could also exist in human subjects between IDO1 activity and combined pain and depression.
Presence of anhedonic behavior exacerbates nociceptive behavior. In order to examine the generality of hippocampal IDO1 expression in relation to the interaction between nociception and depression, we used an established rat model of anhedonia induced by chronic social stress (see Methods). After 2 weeks of chronic social stress, rats 
A

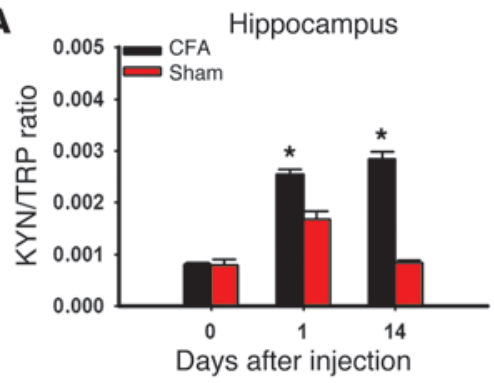

C

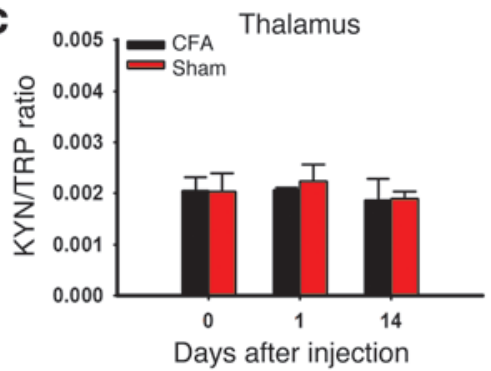

E

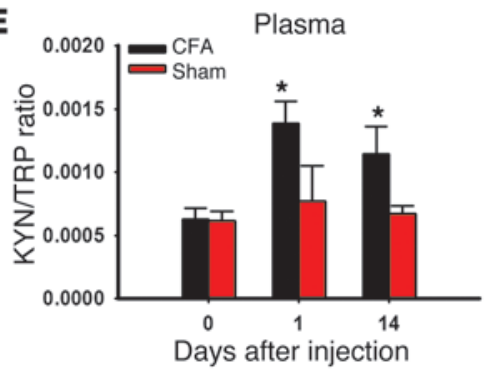

G

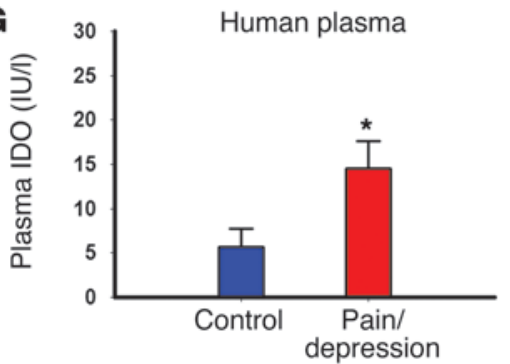

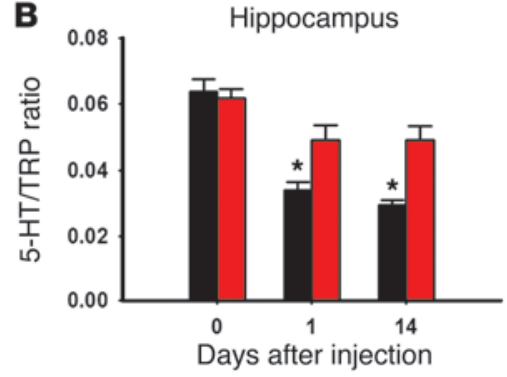
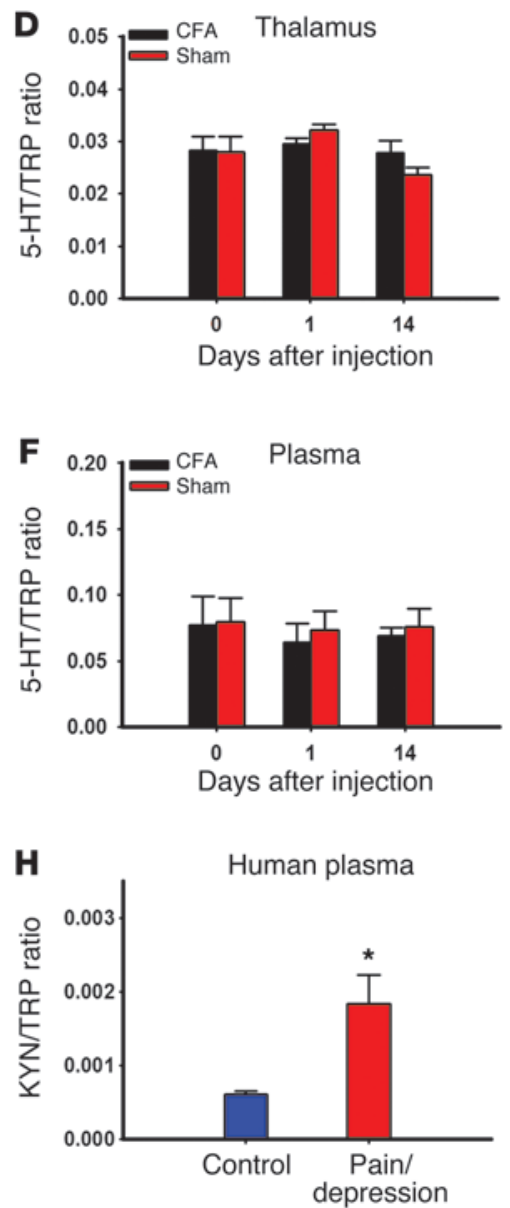

\section{Figure 3}

Altered tryptophan metabolites by IDO enzyme activity. (A and $\mathbf{B}$ ) The kynurenine (KYN)/tryptophan (TRP) ratio was increased (A), whereas the serotonin (5-HT)/TRP ratio was decreased (B), in the contralateral hippocampus of Wistar rats with CFA-induced arthritis as assayed by HPLC. Mean \pm SEM, $n=6,{ }^{*} P<0.05$ compared with sham control. (C and D) The KYN/TRP ratio (C) and the 5-HT/ TRP ratio (D) were not changed in the thalamus of Wistar rats with CFA-induced arthritis as assayed by HPLC. The plasma KYN/TRP ratio (E), but not the 5-HT/TRP ratio $(F)$, was increased in Wistar rats with CFA-induced arthritis when both were examined on day 14. Mean \pm SEM, $n=6,{ }^{*} P<0.05$ compared with sham control. The plasma IDO level (G; ELISA) and the KYN/TRP ratio (H; HPLC), but not the $5-\mathrm{HT} / \mathrm{TRP}$ ratio $(\mathbf{I} ; \mathrm{HPLC})$, were elevated in patients with both chronic back pain and depression. Mean $\pm \mathrm{SEM}, n=13-20,{ }^{*} P<0.05$ compared with healthy control. demonstrated a significant decrease in body weight gain and sucrose preference relative to control rats without social stress (Figure 4A, $F(1,23)=438.20, P<0.05$; Figure $4 \mathrm{~B}, F(1,23)=172.12 ; P<0.05)$, indicating the presence of anhedonic behavior. These rats also exhibited other depressive behaviors, manifesting as a longer immobility time in both FST (Figure 4C; $F(2,35)=31.86, P<0.05$ ) and tail suspension test $(\mathrm{TST}$ ) (Figure 4D; $F(2,35)=369.08, P<0.05)$. Moreover, these same rats exhibited a progressively lower baseline nociceptive threshold in response to mechanical (Figure 4E; $F(1,23)=312,85, P<0.05$ ) and thermal stimulation (Figure $4 \mathrm{~F} ; F(1,23)=100.276, P<0.05$ ) during 3 weeks of chronic social stress, indicating that the presence of anhedonic behavior also influenced baseline nociceptive response.

To examine whether preexisting anhedonic behavior would exacerbate nociceptive behavior following hind paw arthritis, we exposed anhedonic and control rats, following 3 weeks of social stress and sham control respectively, to either CFA hind paw arthritis or sham control and examined behavioral changes 1 week later. Both mechanical allodynia (Figure 4G; $F(4,35)=$ $164.98, P<0.05)$ and thermal hyperalgesia (Figure $4 \mathrm{H} ; F(4,35)=$ $63.72, P<0.05)$ were exacerbated in anhedonic rats as compared with control rats, associated with a significantly longer immobility time in FST (Figure 4I; $F(4,35)=63.19, P<0.05$ ) and TST (Figure 4J; $F(4,33)=73.74, P<0.05)$ in these same rats. Moreover, the IDO1 expression (Western blot) in the bilateral hippocampus was significantly increased in anhedonic rats as compared with control rats with or without hind paw arthritis (Figure 4K; contralateral side only, $P<0.05$ ). These results indicate that nociceptive behavior was exacerbated in rats with preexisting anhedonic behavior, which was also associated with the upregulation of IDO1 expression in the hippocampus. 
A
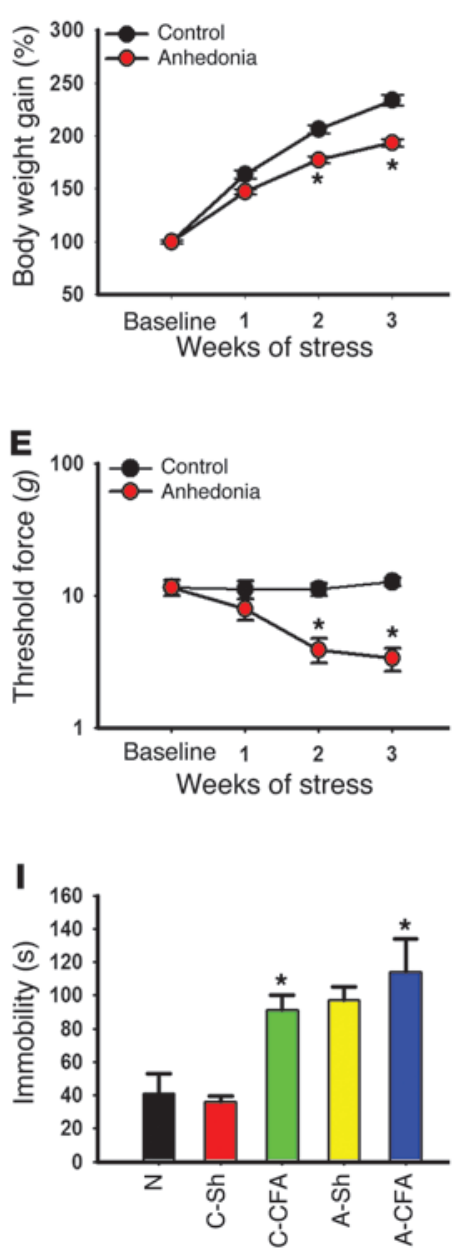

B
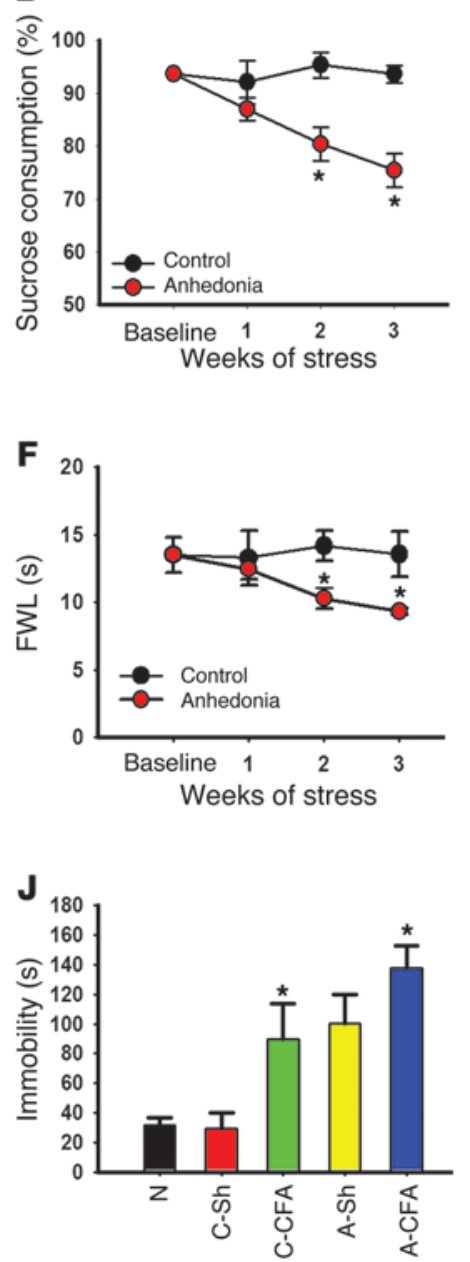

C
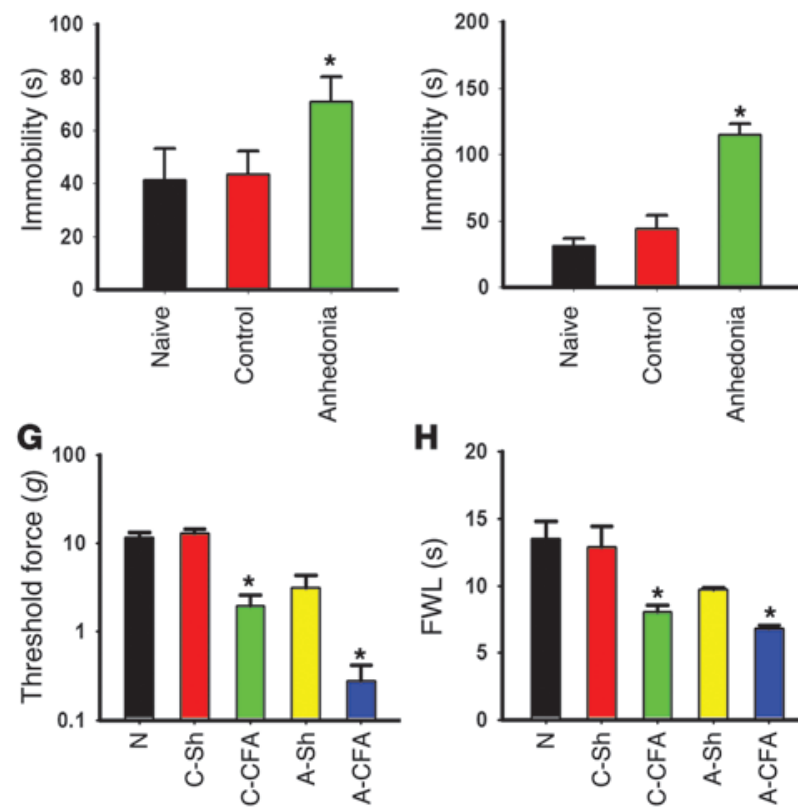

D

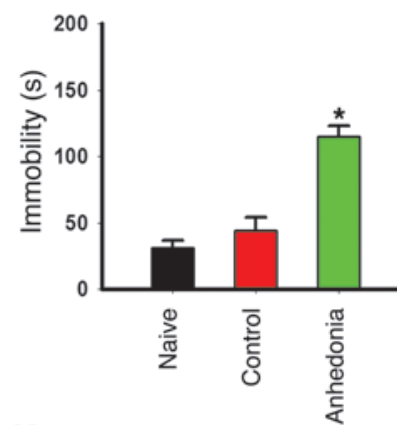

$\mathbf{K}$

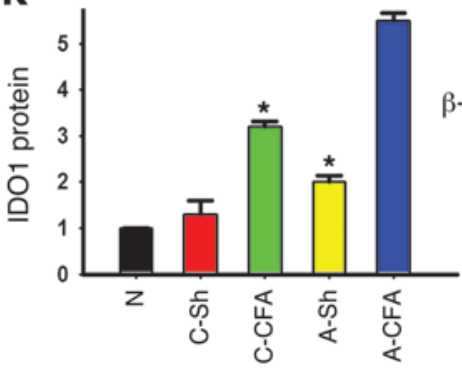

N C-S C-C A-S A-C IDO1 $=-\infty=$

Figure 4

Relationship between anhedonic and nociceptive behavior. (A) The body weight gain was calculated as the percentage of the initial (baseline) body weight. Anhedonic rats gained less weight than control rats. (B) Sucrose preference was calculated as the percentage of the total fluid intake over 24 hours. Sucrose preference was diminished in anhedonic rats. (C-F) The prolonged immobility time in FST (C) and TST (D) was associated with mechanical allodynia (E) and thermal hyperalgesia $(\mathbf{F})$ in anhedonic rats. Data in $\mathbf{A}-\mathbf{F}$ are mean $\pm \mathrm{SEM}, n=6{ }^{*} P<0.05$ compared with control. (G-J) Mechanical allodynia (G) and thermal hyperalgesia (H) were exacerbated, and immobility time was prolonged, in FST (I) and TST $(\mathbf{J})$ in anhedonic rats when examined at 7 days after the CFA-induced monoarthritis. (K) IDO1 protein expression was increased in anhedonic rats (A-Sh and A-CFA) as compared with control rats (C-Sh and C-CFA) with and without CFA injection. Data in $\mathbf{G}-\mathbf{K}$ are mean \pm SEM, $n=6$, ${ }^{\star} P<0.05$ compared with sham control. $\mathrm{N}$, naive.

Inbibition of IDO1 activity concurrently attenuates nociceptive and depressive behavior. To examine whether inhibition of IDO1 activity would influence nociceptive and depressive behaviors in arthritic rats, we administered the IDO1 inhibitor L-1-methyl-tryptophan (1-MT; $10 \mathrm{mg} / \mathrm{d}$ ) (19) or vehicle intraperitoneally twice daily for 14 consecutive days. Treatment with 1-MT, but not vehicle, significantly attenuated both nociceptive (Figure $5 \mathrm{~A} ; F(3,119)=11.33 ; P<0.05)$ and depressive (Figure 5B: $F(3,63)$ $=5.54) ; P<0.05)$ behaviors in arthritic rats. Systemic 1 -MT treatment alone did not alter behaviors in sham controls rats (Figure 5 , A and B), nor did it change the appearance of arthritic hind paw (e.g., redness or swelling).

To examine the brain site of 1-MT action, we microinjected 1-MT ( $5 \mu \mathrm{g}$ in $0.5 \mu \mathrm{l}$ volume, once daily for 7 days) into the hippocampus contralateral to the arthritic hind paw. Intra-hip- pocampal 1-MT treatment also attenuated both nociceptive (Figure 5C; $F(3,71)=5.54, P<0.05$ ) and depressive (Figure 5D; $F(3,31)=14.70, P<0.05)$ behaviors in arthritic rats without changing behaviors in sham control rats, indicating that the hippocampus is a critical brain locus of IDO1 activity. The procedure of brain cannula implantation itself, used for intra-hippocampal microinjection, did not alter the baseline behavioral response when examined 5 days after the surgery (Supplemental Figure 1, B-D).

Intraperitoneal 1-MT treatment also (a) downregulated IDO1 expression (Figure 5E, $P<0.05$ ), (b) lowered the kynurenine/ tryptophan ratio (Figure $5 \mathrm{~F}, P<0.05$ ), and (c) elevated the serotonin/tryptophan ratio (Figure 5G, $P<0.05$ ) in the hippocampus of arthritic rats. Together with the behavioral data, these results indicate that concurrent attenuation of nociceptive and 

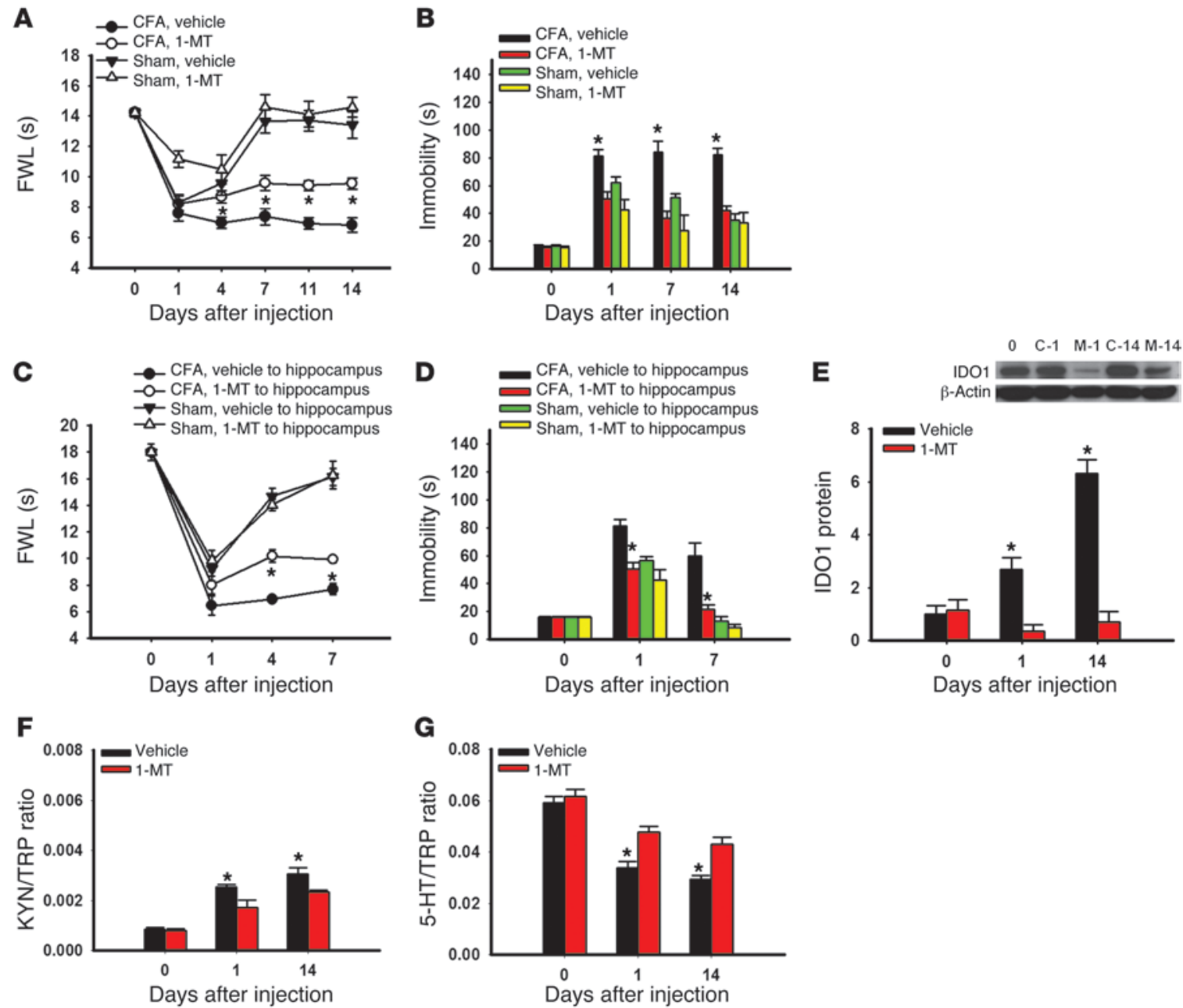

\section{Figure 5}

Effect of IDO1 inhibition on behavioral changes. (A) Intraperitoneal injection of the IDO1 inhibitor 1-MT (10 mg/d), given twice daily for 14 consecutive days beginning immediately after the CFA injection, attenuated mechanical allodynia on the ipsilateral hind paw of rats. (B) The same 1-MT treatment regimen concurrently improved the immobility time in FST in the same rats. Mean $\pm \mathrm{SEM}, n=6$, ${ }^{\star} P<0.05$ compared with vehicle control. (C and D) Intra-hippocampal microinjection of 1-MT (5 $\mu \mathrm{g}$ in $0.5 \mu \mathrm{l})$, once daily for 7 days beginning immediately after the CFA injection, also attenuated thermal hyperalgesia (C) on the ipsilateral hind paw of rats as well as reducing the immobility time in FST (D) in the same rats. Mean \pm SEM, $n=6,{ }^{*} P<0.05$ compared with vehicle control. (E) IDO1 expression in the hippocampus was decreased in rats receiving a 14-day intraperitoneal administration of 1-MT (10 mg/d). Day 0, baseline (naive rats); C-1 and C-14, samples taken on day 1 and day 14 from rats with CFA-induced arthritis; S-1 and S-14, samples taken on day 1 and day 14 from control rats. $\beta$-Actin was used as loading control. Mean \pm SEM, $n=6$, ${ }^{\star} P<0.05$ compared with sham control. The same systemic 1-MT treatment regimen reduced the KYN/TRP ratio $(\mathbf{F})$ and increased the $5-\mathrm{HT} / \mathrm{TRP}$ ratio $(\mathbf{G})$ in the hippocampus. Mean \pm SEM, $n=6,{ }^{*} P<0.05$ compared with vehicle control.

depressive behavior by the 1-MT treatment was mediated by the regulation of hippocampal IDO1 activity, thereby normalizing the content of tryptophan metabolites in the hippocampus.

Ido1 gene knockout attenuates both nociceptive and depressive behavior. To further confirm the role of IDO1 in the behavioral manifestation of pain and depression, we used IDO1-knockout and matched wild-type mice under the same experimental condition as that for Wistar rats. Both basal and arthritis-induced IDO1 expression in the hippocampus, as observed in age-matched wild-type mice, was absent in IDO1-knockout mice (Figure 6A). There were no baseline differences in behavioral tests for nociception (Figure 6, B and C; day 0) and depression (Figure 6, D and E; day 0) between IDO1-knockout and wild-type mice (each $P>0.05$ ). In IDO1- knockout mice, however, both mechanical allodynia (Figure 6B; $F(3,120)=9.86, P<0.01$ ) and thermal hyperalgesia (Figure 6C; $F(3,122)=5.73, P<0.05)$ were significantly attenuated as compared with wild-type mice after the CFA injection into the right tibiotarsal joint. In these same knockout mice, the immobility time (FST) was not increased, nor was there a decrease in the frequency in OFT, as compared with wild-type mice (Figure $6 \mathrm{D} ; F(3,74)=5.40$; Figure $6 \mathrm{E} ; F(3,49)=32.175$, each $P<0.05)$. These results indicate that Ido1 gene knockout concurrently attenuated nociceptive and depressive behavior induced by persistent hind paw nociception.

To examine whether selective reduction of nociceptive behavior would influence depressive behavior and hippocampal IDO1 expression, was given acetaminophen ( $N$-acetyl-para-aminophe- 


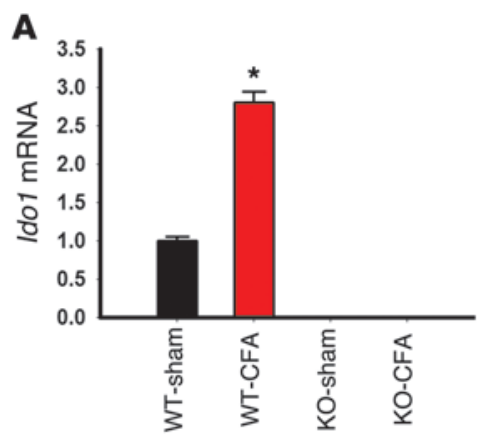

B

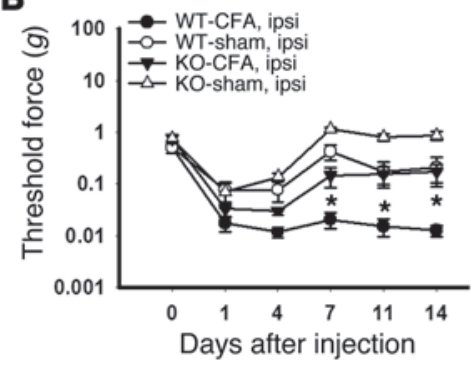

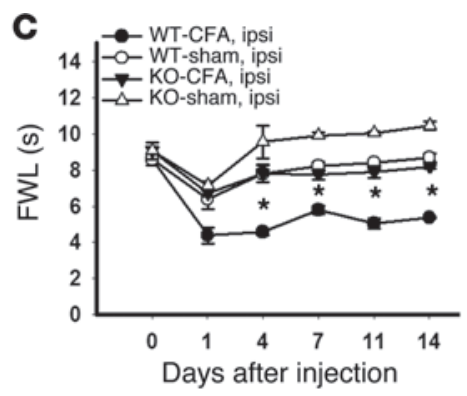

G
$\mathbf{F}$

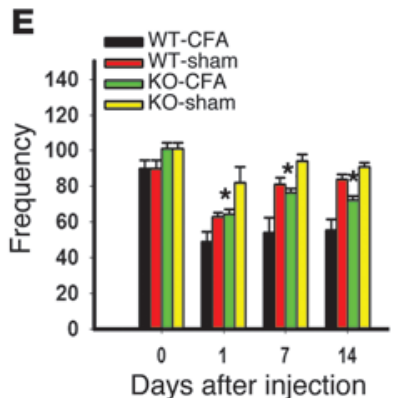

I

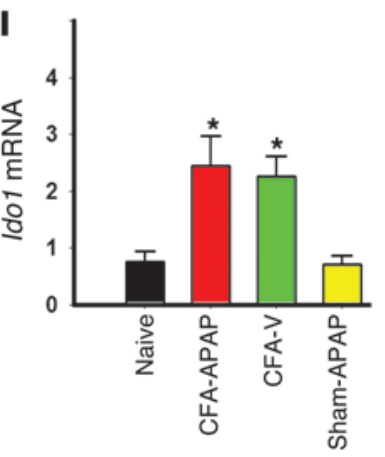

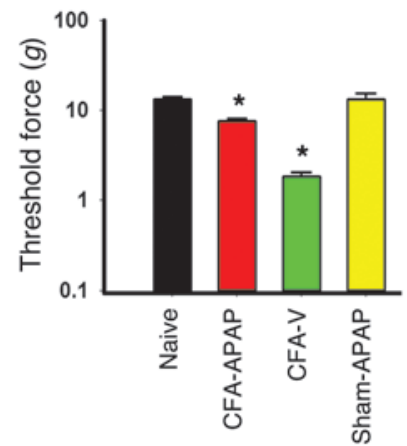

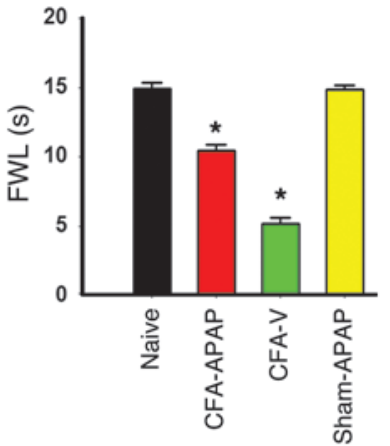

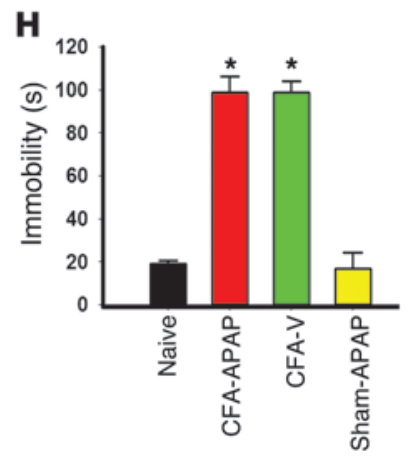

\section{Figure 6}

Effect of IDO-knockout on behavioral changes. (A) IDO-knockout mice had no Ido1 mRNA expression (real-time PCR) in the hippocampus. Ido1 mRNA expression in wild-type mice was increased in the hippocampus after the CFA injection. Mean $\pm \mathrm{SEM}, n=6,{ }^{\star} P<0.05$ compared with sham control. (B and C) Mechanical allodynia (B) and thermal hyperalgesia (C) on the ipsilateral hind paw were attenuated in IDO-knockout mice. (D and E) IDO-knockout also reduced the immobility time in FST (D) and the decreased frequency in OFT (E) in the same mice with CFA-induced arthritis. Mean \pm SEM, $n=6,{ }^{*} P<0.05$ compared with wild-type mice. ( $\mathbf{F}$ and $\mathbf{G}$ ) Intraperitoneal injection of acetaminophen (APAP; $100 \mathrm{mg} / \mathrm{kg}$ ), given once on day 14, attenuated ipsilateral mechanical allodynia (F) and thermal hyperalgesia (G) when mice were examined at 1 hour after the injection. Mean \pm SEM, $n=6,{ }^{*} P<0.05$ compared with vehicle control. (H) The same APAP treatment did not change the immobility time in FST in the same rats. (I) Contralateral hippocampal Ido1 mRNA expression was increased after the CFA injection, which was not reversed by a single APAP treatment. Data in $\mathbf{H}$ and $\mathbf{I}$ are mean \pm SEM, $n=6,{ }^{*} P<0.05$ compared with vehicle control.

nol, $100 \mathrm{mg} / \mathrm{kg})(20,21)$, an analgesic agent without the anti-inflammatory effect, or vehicle once intraperitoneally on day 14 to arthritic or sham rats. When examined at 1 hour after the treatment, acetaminophen, but not vehicle, significantly reduced mechanical allodynia (Figure $6 \mathrm{~F} ; F(3,23)=128.80, P<0.05)$ and thermal hyperalgesia (Figure $6 \mathrm{G} ; F(3,23)=839.97, P<0.05$ ). The acetaminophen treatment did not acutely reverse depressive behavior (Figure 6H; FST), nor did it alter the Ido1 mRNA level in the same arthritic rats (Figure 6I). These results indicate that the correlation between nociception and depression demonstrated in these rats was not a simple coincidence but rather that the two were linked by the hippocampal IDO1 expression.
IL-6 and JAK/STAT are increased in rats with nociceptive and depressive behavior. Proinflammatory cytokines including IL-6 have been shown to be involved in the cellular mechanisms of both pain and depression $(11,12,22)$. To examine the hypothesis that proinflammatory cytokines such as IL- 6 and one of its downstream signaling pathways (JAK and STAT) $(23,24)$ would mediate hippocampal IDO1 upregulation, we first examined whether the IL-6 level and JAK/STAT expression would be increased in rats with coexistent nociceptive and depressive behavior. Both the plasma IL-6 level and hippocampal Il6 mRNA expression were significantly increased in rats with nociceptive and depressive behavior as compared with sham rats (Figure 7, A and B; $P<0.05$ ). The hippocampal Il6 mRNA 


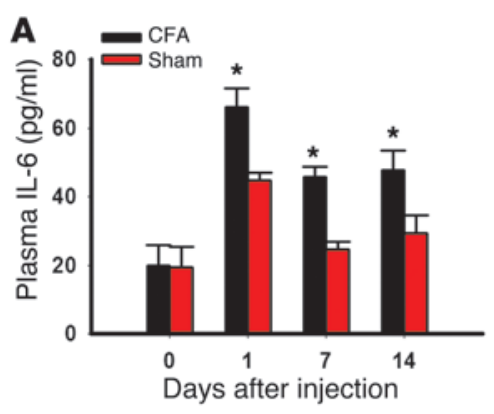

E

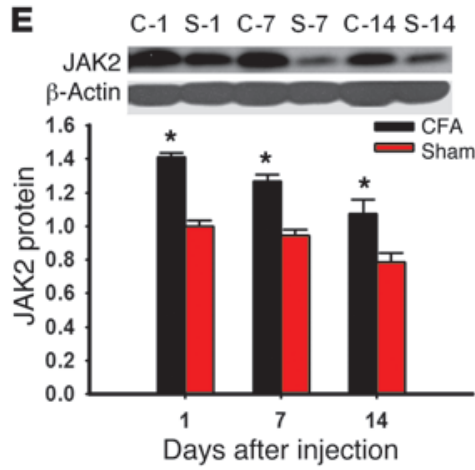

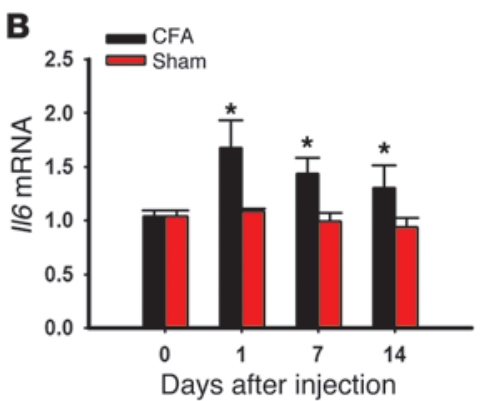

C

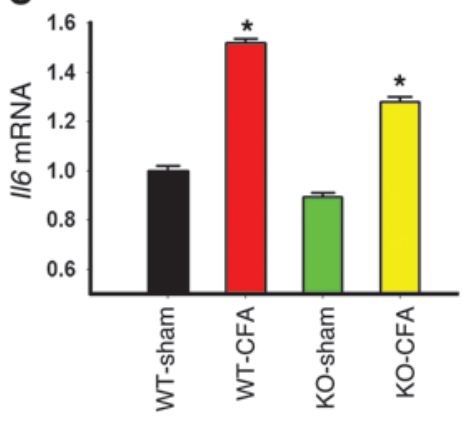

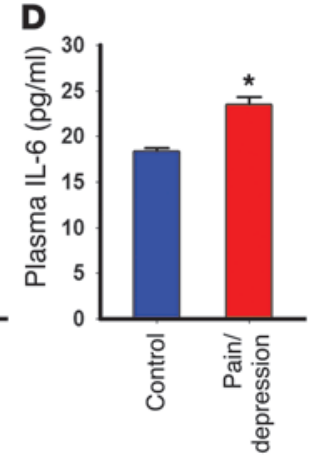
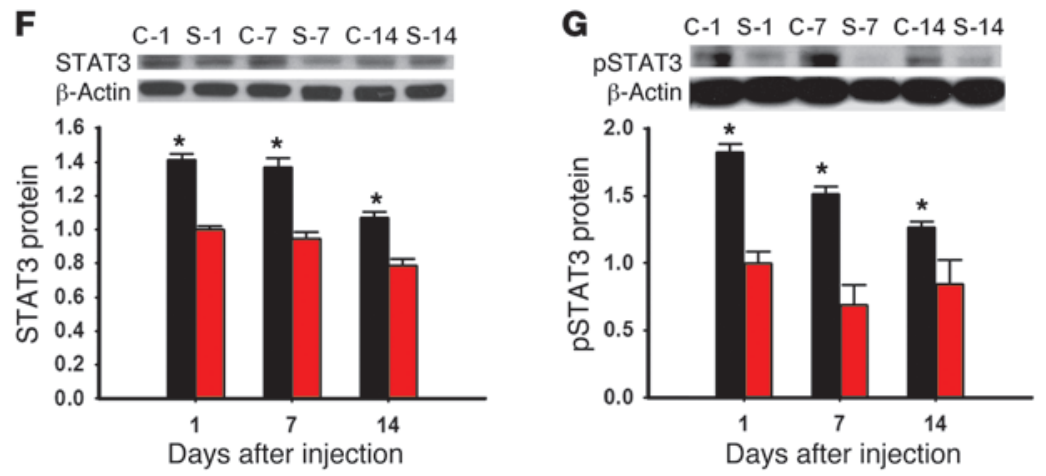

\section{Figure 7}

Role of IL-6 in the hippocampal JAK/STAT pathway. (A and B) The plasma IL-6 concentration was increased (A, ELISA), as was I/6 mRNA expression in the hippocampus (B, real-time PCR), in rats with CFA-induced arthritis. (C) //6 mRNA expression was also increased in the hippocampus of both IDO-knockout and wild-type mice with CFA-induced arthritis. Mean $\pm \mathrm{SEM}, n=6,{ }^{*} P<0.05$ compared with sham control. (D) The plasma IL-6 level was elevated in patients with both chronic back pain and depression (ELISA). Mean \pm SEM, $n=13-20,{ }^{*} P<0.05$ compared with healthy control. (E-G) The expression of JAK2 (E), STAT3 (F), and p-STAT3 $(\mathbf{G})$ in the hippocampus was increased in rats with CFA-induced arthritis (Western blot analysis). C-1, C-7, and C-14, samples taken on days 1, 7, and 14 from rats with CFA-induced arthritis; S-1, S-7, and S-14, samples taken on days 1, 7, and 14 from sham control rats. $\beta$-Actin was used as loading control. Mean \pm SEM, $n=4-5$, ${ }^{*} P<0.05$ compared with sham control.

level was also elevated in IDO1-knockout and wild-type mice after CFA injection into a tibiotarsal joint (Figure 7C; $P<0.05$ ), indicating that the IL- 6 increase was upstream of IDO1 upregulation. In patients with both chronic pain and depression, the plasma IL-6 content was also elevated as compared with that in healthy control subjects (Figure 7D; $P<0.05$ ). Of note, plasma IL-6 content in human subjects was measured in a cross-sectional observational setting and could have been influenced by the subjects' underlying pain condition and other variations such as body weight. Moreover, the expression of IL-6 signaling elements, including JAK2, STAT3, and P-STAT3, was all elevated in the hippocampus of rats with nociceptive and depressive behavior as compared with sham controls (Figure 7, E-G; each $P<0.05$ ).

IL-6 induces in vitro IDO1 upregulation. To examine a direct relationship between IL-6 and IDO1 expression at the cellular level, we exposed cultured Neuro2a cells to exogenous IL- $6(0.5 \mathrm{ng} / \mathrm{ml})$ or vehicle (PBS) for 24 hours. IDO1 immunoreactivity was detected in the perinuclear cytoplasm of Neuro2a cells and increased following exposure to IL-6 for 24 hours (Figure 8A). Exposure of cultured Neuro2a cells to exogenous IL-6, but not vehicle, significantly increased Ido1 mRNA (real-time PCR) and protein (Western blot analysis) expression (Figure 8, B and C; $P<0.05$ ), resulting in the increased kynurenine/tryptophan ratio and decreased serotonin/tryptophan ratio (HPLC) in these Neuro2a cells (Figure 8, D and E; $P<0.05$ ).
Furthermore, we used a hippocampal organotypic slice culture taken from postnatal rats to examine the in vitro effect of IL-6 on hippocampal IDO1 expression and activity. After being cultured for 1 week, hippocampal slices were treated with IL-6 (100 $\mathrm{ng} / \mathrm{ml}$ ) or vehicle (PBS) for 24 hours. Exposure of exogenous IL-6, but not vehicle, increased IDO1 immunoreactivity (Figure 8F) and upregulated the expression of Ido1 mRNA (real-time PCR, Figure 8G; $P<0.05$ ) and protein (Western blot analysis, Figure $8 \mathrm{H}$; $P<0.05)$ in cultured slices. Under the same experimental condition, the kynurenine/tryptophan ratio was significantly increased, whereas the serotonin/tryptophan ratio was decreased in the culture medium (HPLC, Figure 8I; $P<0.05$ ). Collectively, the results indicate that IL- 6 has a direct cellular effect on IDO1 expression in the hippocampus.

IL-6-mediated hippocampal IDO1 expression concurrently regulates nociceptive and depressive behavior. To examine the functional role of IL-6 signaling in hippocampal IDO1 expression as well as its contribution to both nociceptive and depressive behavior, we microinjected an IL- 6 antiserum into the hippocampus of arthritic or sham control rats. Microinjection of IL-6 antiserum $(0.5 \mu \mathrm{g}$, once daily for 7 days) (22), but not control serum (vehicle), into the hippocampus contralateral to arthritic hind paw significantly attenuated mechanical allodynia (Figure 9A; $F(1,39)$ $=9.28, P<0.05)$, thermal hyperalgesia (Figure 9B; $F(1,39)=7.46$, 
A

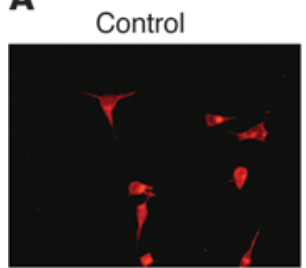

IDO1

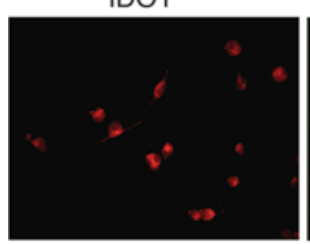

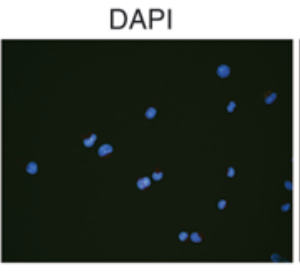
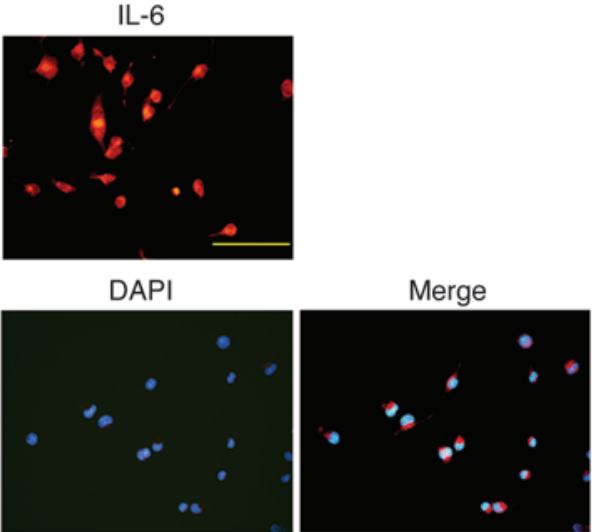

DAPI
B

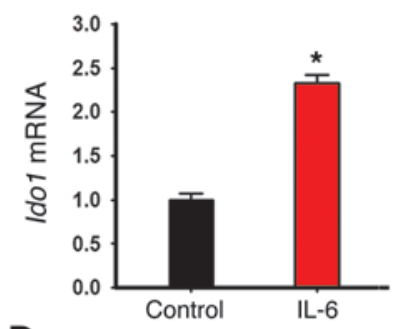

D

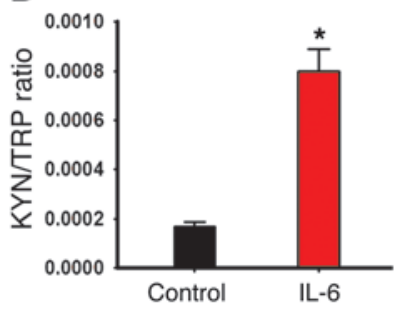

C

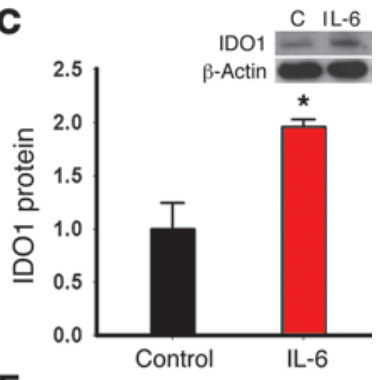

E

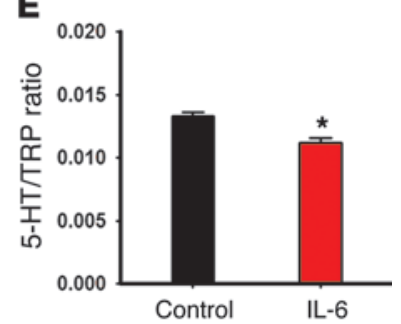

F
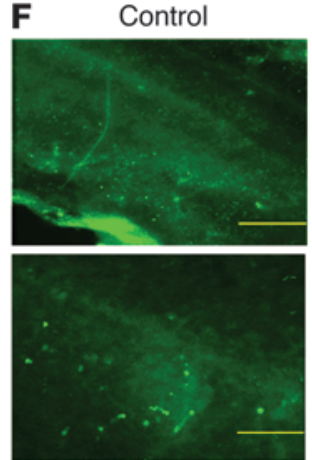

IL-6

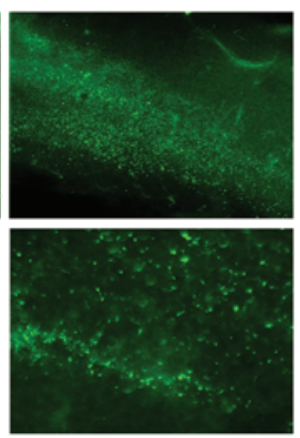

G

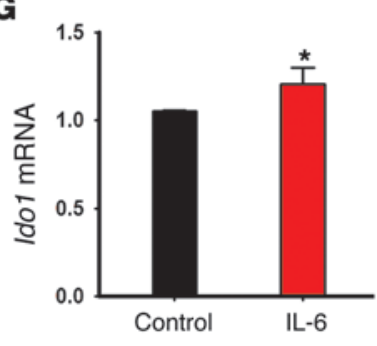

I

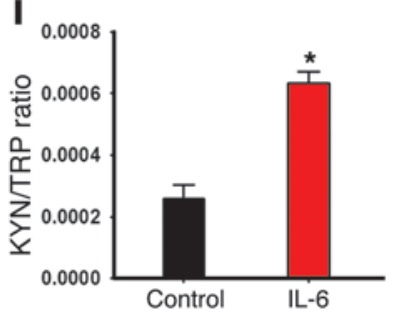

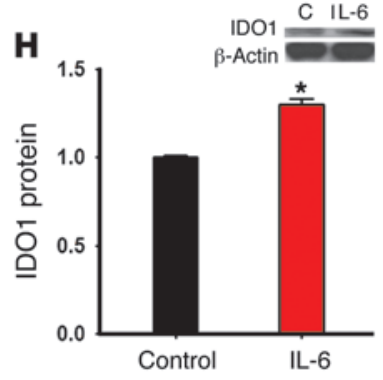

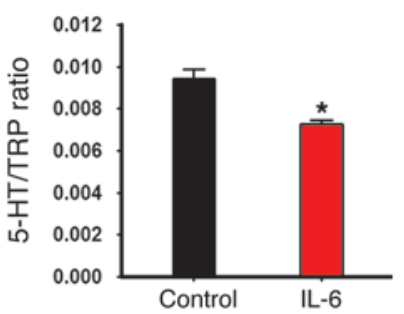

Figure 8

Effects of IL-6 on IDO1 expression in Neuro2a cells and organotypic hippocampal culture. (A) IDO1 immunoreactivity was increased in IL-6treated Neuro2a cells, as expressed in perinuclear cytoplasm when samples were costained with DAPI. Scale bar: 50 um. (B-E) IDO1 mRNA (B) and protein (C) expression, as well as IDO activity (KYN/TRP ratio [D] and 5-HT/TRP ratio [E]), was increased in cultured Neuro2a cells after addition of IL-6 $(0.5 \mathrm{ng} / \mathrm{ml})$ for 24 hours. (F-H) Exposure of exogenous IL-6 $(100 \mathrm{ng} / \mathrm{ml})$ for 24 hours increased the expression of IDO1 (F: immunoreactivity; G: mRNA; H: Western blot) in hippocampal organotypic slice culture. Scale bars: $500 \mu \mathrm{m}$ (top row) and $50 \mu \mathrm{m}$ (bottom row). C, control. In addition, adding IL-6 for 24 hours also increased the KYN/TRP ratio and decreased the 5-HT/TRP ratio (HPLC) in the culture medium (I). ${ }^{*} P<0.05$ compared with vehicle control.

$P<0.05$ ), and depressive behavior (Figure 9C; $F(3,19)=155.99$, $P<0.001)$. The same IL-6 antiserum treatment also prevented IDO1 upregulation in the hippocampus (Figure 9D; $P<0.05$ ), consistent with the in vitro results of IL-6-induced IDO1 expression (Figure $8, \mathrm{~B}$ and $\mathrm{C}$ ).

Conversely, microinjection of exogenous IL-6 (recombinant rat IL-6, $0.1 \mu \mathrm{g}$, once daily for 7 days) (25), but not vehicle, into the left hippocampus of naive rats (without arthritis) induced right hind paw mechanical allodynia (Figure 9E: $F(3,79)=2.54, P<0.05$ ) and thermal hyperalgesia (Figure 9F: $F(3,103)=11.24, P<0.01$ ), as well as depressive behavior (Figure 9G: $F(3,19)=65.20, P<0.001$ ) and increased Ido1 mRNA expression in the hippocampus (Figure 9H; $P<0.05$ ). These IL-6 effects were prevented when IL-6 was co-administered with the JAK/STAT inhibitor AG490 (5 $\mu \mathrm{g}$, once daily for 7 days) (26) into the hippocampus (Figure 9, E-H; each $P<0.05)$. Intra-hippocampal microinjection of AG490 alone had no effect on the baseline behavioral response and Ido1 mRNA expression in naive rats (Figure 9, E-H; each $P>0.05$ ).

Taken together with the data obtained using the IDO1 inhibitor 1-MT, these findings indicate that the hippocampus is a central site of IL-6-regulated IDO1 expression critically contributory to the comorbid interaction between pain and depression. 
A
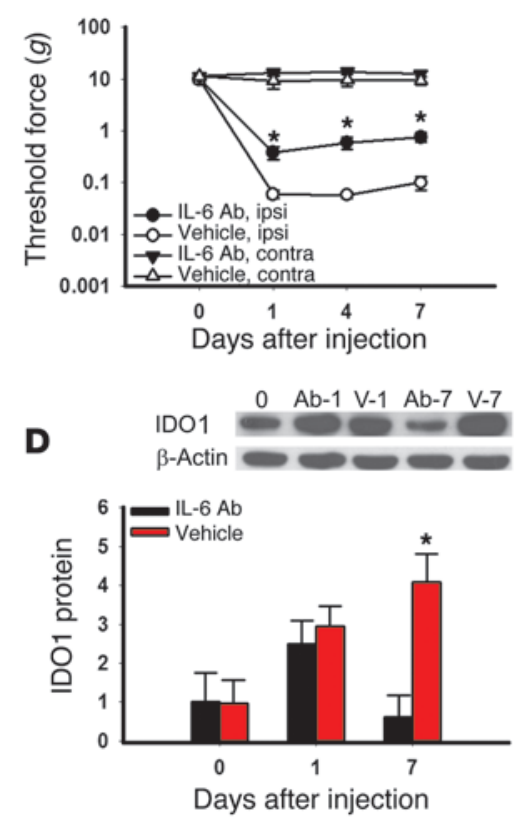

G

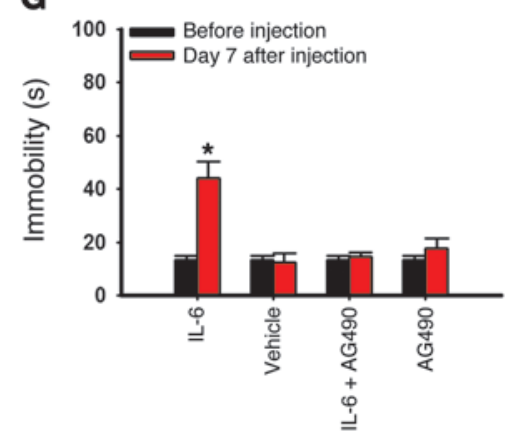

B
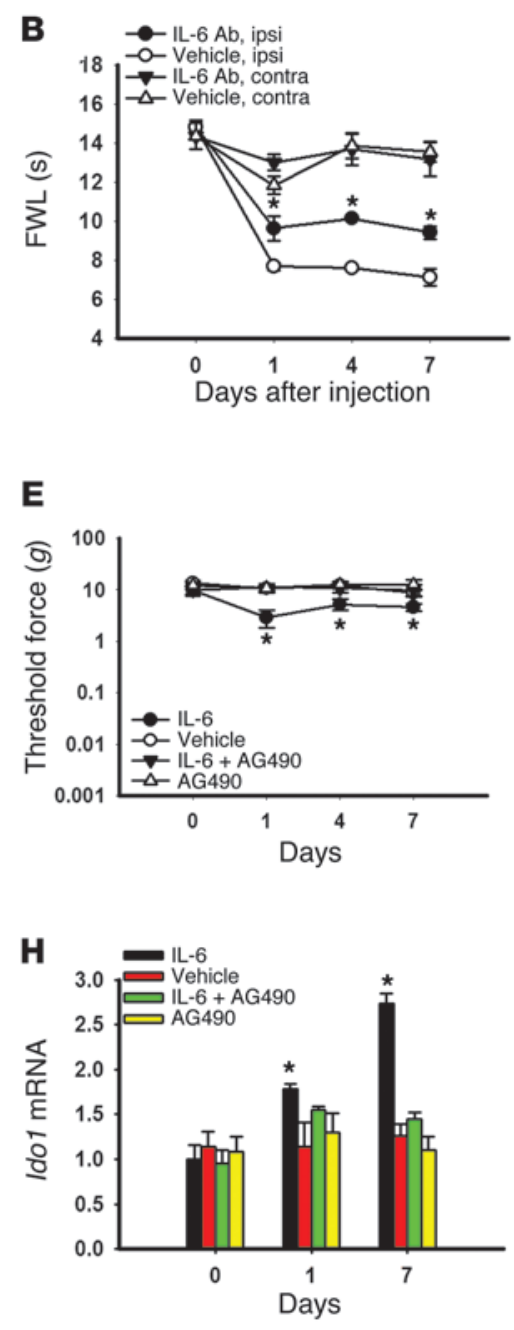

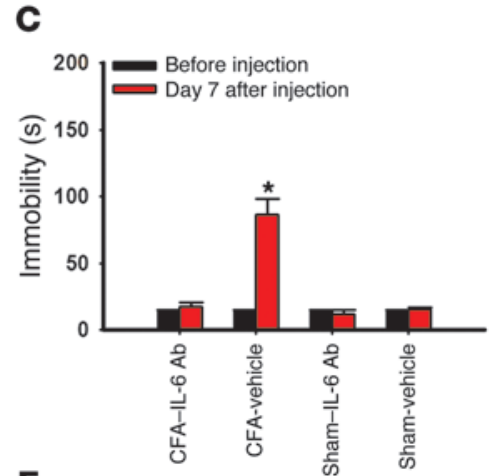

$\mathbf{F}$

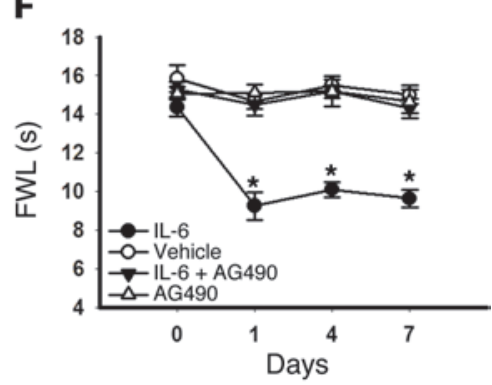

Figure 9

Role of IL-6 signaling in hippocampal IDO expression and behavioral changes. (A-D) Microinjection of an IL-6 antiserum (IL-6 Ab; $0.5 \mu \mathrm{g} / \mathrm{d}$; once daily for 7 days) into the hippocampus contralateral to the hind paw with CFA-induced arthritis attenuated mechanical allodynia (A) and thermal hyperalgesia (B) on the ipsilateral hind paw, without a change in nociceptive threshold in sham rats (data not shown). The same IL-6 antiserum microinjection regimen concurrently improved depressive behavior in FST (C) and prevented the hippocampal IDO1 upregulation (D) in these same rats. Day 0 , baseline (naive rats); $A b-1$ and Ab-7, samples taken on day 1 and day 7 from Wistar rats treated with IL- 6 antiserum; $V-1$ and $\mathrm{V}$-7, samples taken on day 1 and day 7 from Wistar rats treated with control serum. $\beta$-Actin was used as loading control. Mean \pm SEM, $n=6$, ${ }^{*} P<0.05$ compared with vehicle (control serum). (E-H) Microinjection of exogenous IL-6 $(0.1 \mu \mathrm{g} / 0.5 \mu \mathrm{l}$; once daily for 7 days) into the left hippocampus of naive rats (without CFA injection) induced mechanical allodynia (E) and thermal hyperalgesia (F) on the right hind paw. The same IL-6 microinjection regimen concurrently induced depressive behavior in FST (G) and upregulated hippocampal Ido1 mRNA expression (H) in these same rats. The effects from the intra-hippocampal microinjection of IL-6 were blocked when IL-6 was co-administered with AG490 (a JAK/STAT inhibitor; $5 \mu \mathrm{g} / 0.5 \mu \mathrm{l})$ for 7 days. Mean $\pm \mathrm{SEM}, n=4-5,{ }^{*} P<0.05$ compared with vehicle control.

\section{Discussion}

We have demonstrated that IDO1 expression was selectively upregulated in the hippocampus of Wistar rats with coexistent nociceptive and depressive behavior. The IDO1 level was also elevated in patients with both pain and depression. Either Ido1 gene knockout or inhibition of IDO1 activity, but not a transient reversal of nociceptive behavior alone by acetaminophen, concurrently attenuated nociceptive and depressive behavior. At the cellular level, the hippocampal IDO1 expression was mediated through IL-6 and its downstream JAK/STAT signaling pathway, which in turn altered the kynurenine/tryptophan and serotonin/tryptophan ratios in the hippocampus. The results indicate that brain IDO activity played a critical role in regulating the comorbid interaction between nociceptive and depressive behaviors.

A comorbid relationship between pain and depression has long been recognized in the clinical setting $(27,28)$. Earlier studies focused on a temporal relationship between pain and depression (e.g., antecedent hypothesis versus consequence hypothesis) $(29,30)$, but the cellular mechanism underlying this relationship remains unknown. Recent neurobiological studies have suggested 
that both depression and chronic pain may involve the monoaminergic system, the hypothalamic/pituitary/adrenal axis, as well as various other neurotransmitters/neuromodulators including acetylcholine, GABA, substance P, cholecystokinin, endogenous opioid, and brain-derived neurotrophic factor (31-35). Despite some progress, clinical treatment of pain and depression has so far been limited to symptomatic management $(3,36)$. A number of studies have also suggested that the effect of antidepressants on chronic pain is not necessarily related to their anti-depression property (36-38).

In the present study, the data from human subjects suggests an apparent relationship between IDO expression/enzyme activity and clinical symptoms of pain and depression, but this cross-sectional clinical observation does not explore the causal relationship between IDO and clinical conditions. On the other hand, the data from animal experiments suggest a novel mechanistic link between pain and depression via a critical role of IDO1 in the hippocampus. Regulation of hippocampal IDO1 is likely mediated through an IL- 6 signal transduction pathway, because (a) upregulation of IL-6 as well as downstream JAK and STAT3 preceded the IDO expression in rats with combined nociceptive and depressive behavior; (b) the hippocampal Il6 mRNA level was elevated in Ido1 gene knockout mice in response to inflammatory arthritis; (c) inhibition of IDO1 activity by systemic 1-MT treatment did not prevent elevation of the plasma IL-6 level in rats with both nociceptive and depressive behavior (data not shown); and (d) IL-6 directly upregulated IDO1 expression in both Neuro2a cells and an organotypic hippocampal tissue culture. Our data also indicate that the hippocampus is a critical brain region of IDO regulation, because IDO1 was selectively upregulated in the hippocampus, but not in the thalamus or nucleus accumbens. These findings are consistent with the previous reports that (a) certain brain regions including the hippocampus play a critical role in the integration of mood changes and pain $(31,39,40)$ and (b) the hippocampus is related to nociceptive perception and its exacerbation by mood disorders such as anxiety $(41,42)$.

Kynurenine and serotonin are two major tryptophan metabolites produced via enzymatic regulation including IDO, which have been implicated in the mechanisms of pain and depression $(4,5)$. Our data indicate that both kynurenine/tryptophan and seroto$\mathrm{nin} /$ tryptophan ratios in the hippocampus were closely regulated by IDO1 activity. This regulatory mechanism appears to have two important functional implications: on the one hand, increased IDO activity lowers the endogenous serotonin level (43), which leads to depression (44) and diminishes the descending inhibition of pain modulation $(45,46)$; on the other hand, increased IDO activity increases kynurenine derivatives such as quinolinic acid, contributing to neurotoxicity and nociception via the interaction with glutamate receptors (47). Therefore, IDO is situated in a key tryptophan metabolic pathway, and alteration of IDO activity results in changes in the content of endogenous kynurenine and serotonin, both of which play a critical role in the mechanisms of pain and depression (Supplemental Figure 2). This notion is supported by our data showing that concurrent improvement of pain and depression was achieved by inhibiting IDO1 activity or IDO gene knockout, which normalized the increased kynurenine/ tryptophan ratio and decreased the serotonin/tryptophan ratio resulting from hippocampal IDO upregulation. It would be of interest in future studies to examine the relationship between IDO expression and other products of tryptophan metabolism and its role in pain and depression.
Studies in the immunology field have consistently shown a relationship between inflammatory mediators and IDO expression in immune cells $(48,49)$. Studies using central administration of cytokines have indicated a role for cytokines in various behavioral manifestations. For example, intracerebroventricular (i.c.v.) administration of IL- 6 or IL-1 $\beta$ elicited hyperalgesia (50), as well as fever, anorexia, and reduction of social exploratory behavior (51, 52). Three recent studies including ours have shown that peripheral nerve injury induced depressive behavior in rats (53-55), which is associated with an increased IL- $1 \beta$ expression in the frontal cortex (54). The present data demonstrate a direct link between cytokine signaling and IDO expression in the hippocampus. Given that IDO changes were also demonstrated in a rat model of anhedonia, regulation of brain IDO expression may have a broad implication in the interaction between pain and depression. It will be of considerable interest in future studies to determine whether a similar cytokine and IDO link would be relevant to other pain conditions such as neuropathic pain.

The present study supports a central (hippocampal) effect of IL-6-mediated IDO activity on the behavioral interaction between pain and depression. First, intra-hippocampal microinjection of the IDO1 inhibitor 1-MT attenuated both nociceptive and depressive behavior similar to that after systemic 1-MT administration. Second, neither systemic 1-MT nor intra-hippocampal administration of IL-6, IL-6 antiserum, or 1-MT changed signs of hind paw inflammation (e.g., redness, swelling), suggesting that the effect of 1-MT on nociceptive and depressive behavior is unlikely to be mediated through a peripheral mechanism at the site of hind paw arthritis. Third, the plasma IDO activity, reflected by an increased kynurenine/tryptophan ratio, was only transiently increased on day 1 but not day 7 and 14 after hind paw inflammation. Fourth, exogenous IL-6 directly upregulated IDO1 expression and enhanced IDO activity in Neuro2a cells and an organotypic hippocampal tissue culture. Fifth, intra-hippocampal microinjection of IL-6 in naive rats induced hippocampal IDO upregulation as well as nociceptive and depressive behavior, which was blocked by AG490 (a JAK/STAT inhibitor). Thus, converging evidence from immunology literature and the current study suggests an important role of IDO activity in the central nervous system in addition to its critical role in immunoregulation (56).

Clinical studies have demonstrated that the plasma IL-6 level was increased in patients with painful neuropathy, cancer, inflammation $(57,58)$, and depression $(59,60)$. In this study, the plasma IL- 6 and IDO level, as well as IDO enzyme activity, was increased in patients with chronic back pain and depression, consistent with the findings from animal studies. This raises the possibility that concurrent treatment of both pain and depression might be possible through regulation of brain IDO activity, in contrast to the current approach of symptomatic management using antidepressants and analgesics (3). Although the neural and cellular mechanism underlying the interaction between pain and depression is likely to be complex and involves other neurotransmitters and neuromodulators (25-33), the present findings may suggest a new strategy of clinical intervention. This new strategy focuses on both prevention and reversal of comorbid interactions between pain and depression by targeting its underlying mechanism involving altered ratios of endogenous tryptophan metabolites resulting from upregulated IDO expression in certain brain regions. Since IDO inhibitors have been tried in clinical studies of depression and cancer treatment (61-63), it will be of considerable interest 
to examine whether using IDO inhibitor, alone or in combination with other agents blocking IDO upregulation or regulating tryptophan metabolism, would be able to achieve concurrent alleviation of pain and depression in the clinical setting.

\section{Methods}

\section{Human subjects}

Male and female subjects between 18 and 65 years old were recruited. A total of 33 subjects were recruited from the greater Boston area. Inclusion criteria included (a) chronic lumbar or cervical radicular pain due to disk herniation or radiculitis for at least 3 months; (b) clinical depression without hospitalization within the past 12 months or medication changes; (c) no history of drug abuse confirmed by a negative urine drug screen; (d) no other psychiatric disorders, including schizophrenia, bipolar disorder, and eating disorders; and (e) for normal control subjects, no pain or depression for at least the past 6 months. Board-certified physicians on the research team reviewed and confirmed clinical diagnosis of pain and depression. Blood samples were taken once and used for HPLC and ELISA assays. Of note, the data from human subjects was obtained in a cross-section observational setting, which could be confounded by potential clinical variations such as body weight and a subject's underlying pain condition as well as a small sample size.

\section{Experimental animals}

Male Wistar rats (250-300 g; Charles River) or 7- to 10-week-old male B6.129-Ido $1^{\mathrm{tm} 1 \mathrm{Alm}} / \mathrm{J}\left(\mathrm{IDO}^{-/-}\right)$and C57BL/6J wild-type mice (Jackson Laboratory) were used. Animals were housed individually $\left(21 \pm 2{ }^{\circ} \mathrm{C}\right.$, relative humidity $50 \% \pm 10 \%, 12$-hour light/12-hour dark cycle), with water and food available ad libitum.

\section{Surgical procedures}

Hind paw monoarthritis. Hind paw monoarthritis was induced by the injection of CFA $(50 \mu \mathrm{l})$ into a unilateral tibiotarsal joint cavity under brief isoflurane anesthesia (13). Animals in sham groups were injected with $50 \mu \mathrm{l}$ incomplete Freund's adjuvant. Local redness and joint swelling were observed in CFA-injected rats but not control rats during the experimental period.

Brain cannula implantation and drug injection. Under brief isoflurane anesthesia, a guide cannula (26 gauge, Plastics One) was implanted just above the hippocampus (anteroposterior [AP]: $-3.6 \mathrm{~mm}$; left: $+2.0 \mathrm{~mm}$ from bregma; depth: $-4.0 \mathrm{~mm}$ from skin) (64). An injection needle (33 gauge, Plastics One) was inserted through the guide cannula, and drug solution or vehicle $(0.5 \mu \mathrm{l})$ was slowly injected over 5 minutes using a Hamilton syringe. Locations of the cannula placement were confirmed at the time of tissue harvest (Supplemental Figure 3A).

\section{Anhedonia induced by chronic social stress}

To induce anhedonia-like behavior in rats, chronic social stress was introduced using a modified resident-intruder social interaction method as described previously (65-67). An experimental rat (275-300 g), designated as an intruder rat in this model, was transferred from its home cage into a cage of a resident rat (500-600 g) for 1 hour per day. The intruder rat and the resident rat were separated by a small, round wiremesh compartment (diameter $11 \mathrm{~cm}$, height $14 \mathrm{~cm}$ ) within the resident cage. After 1 hour, the intruder rat was returned to its home cage. This procedure was carried out at the beginning of the dark cycle in a 24-hour light cycle (light on and off for each 12 -hour period). An intruder rat was confronted with a different resident rat each day. This process lasted for 4 weeks. For controls, rats were placed in the same behavioral room but without the social interaction with a resident rat.

\section{Behavioral tests}

Nociceptive test. Animals were habituated to a test setting (30-minute session) for 3 consecutive days. Thermal withdrawal threshold was assessed using the Hargreaves apparatus and method (68). Mechanical withdrawal threshold was assessed using von Frey filaments (69).

FST. FST was performed according to the method of Porsolt et al. (14). The total duration of immobility (non-swimming) within a 5-minute session was recorded as immobility scores (in seconds) and compared among groups.

TST. TST was carried out as described previously (70). Briefly, a rat was hung on a hook, using adhesive tape placed $20 \mathrm{~mm}$ from the tip of its tail, on a 50 -cm-height rod. The immobility time during a 6 -minute period of tail suspension was recorded with a stopwatch.

OFT. OFT was carried out in a Plexiglas square box $(57 \times 57 \mathrm{~cm})$ with walls $50 \mathrm{~cm}$ in height (71). Behaviors were observed for 10 minutes under a dim light. After the first 5 minutes (habituation), the number of squares crossed by a rat was recorded for the next 5 minutes.

Sucrose preference test. A sucrose preference test (SPT) was performed as described in previous studies $(72,73)$. To perform this test, $1 \%$ sucrose solution was offered once weekly in a rat's home cage. Each rat had a free choice between $1 \%$ sucrose solution bottle and a tap water bottle. Sucrose and tap water intakes were separately measured by weighing each bottle before and after the test (a 24-hour period). The sum of tap water and sucrose water intake (in grams) was calculated as the total water intake. The sucrose preference was expressed as the percentage of sucrose water intake relative to the total water intake.

Rotarod test. A rotarod test, using an accelerating rotarod apparatus (Columbus Instruments), was performed in both arthritic and sham control rats. Rats were given 3 training sessions and then placed on a 9-cm-diameter rod with speed increased from 0.5 to $30 \mathrm{rpm}$ over a 60 -second period. Each rat was tested in 3 consecutive trials with 15 -minute intervals. The duration of time on the rotarod was determined automatically by a timer that recorded to the nearest second.

\section{Cultures}

Neuro2a cell culture. Neuro2a cells were cultured in Eagle's minimal essential medium (Invitrogen) supplemented with $10 \%$ fetal bovine serum, $100 \mathrm{U} / \mathrm{ml}$ penicillin, and $100 \mu \mathrm{g} / \mathrm{ml}$ streptomycin (Invitrogen) in a humidified atmosphere of $5 \% \mathrm{CO}_{2}$ and $95 \%$ air at $37^{\circ} \mathrm{C}$. Cells were treated with $0.5 \mathrm{ng} / \mathrm{ml}$ IL-6 or vehicle and collected 24 hours later for assays (74).

Organotypic hippocampal tissue culture. According to a modified method $(75,76)$, organotypic hippocampal cultures were prepared using hippocampus slices from 5- to 8-day-old Wistar rats. Rat pups were euthanized by decapitation, brains were rapidly removed, and the hippocampus was separated from the brain. Hippocampal slices ( $350 \mu \mathrm{m}$ in thickness) were made using a McIlwain tissue chopper (Campden Instruments Ltd.) and placed in Gey's balanced salt solution (Sigma-Aldrich) with $2 \mathrm{mg} / \mathrm{ml} \mathrm{D-glu-}$ cose. Slices were then placed on 30-mm Millicell-CM (Millipore) porous $(0.4 \mu \mathrm{m})$ membranes. These membranes were transferred into 6-well culture plates filled with an incubation medium $(1 \mathrm{ml})$ consisting of $50 \% \mathrm{vol} /$ vol minimal essential medium, 25\% heat-inactivated horse serum, and $25 \%$ HBSS (Invitrogen), supplemented with $25 \mathrm{mM}$ D-glucose. Plates were incubated in a humidified atmosphere of $5 \% \mathrm{CO}_{2}$ and $95 \%$ air at $37^{\circ} \mathrm{C}$. Culture medium was changed 3 times a week. Slices were treated with $100 \mathrm{ng} / \mathrm{ml}$ IL-6 or vehicle and collected 24 hours later for assays (77).

\section{Immunohistochemistry}

Rats were anesthetized with pentobarbital ( $50 \mathrm{mg} / \mathrm{kg}$, intraperitoneally) and perfused transcardially with cold $0.9 \%$ saline ( $\mathrm{pH} 7.4 \pm 0.1$ ), followed by cold $4 \%$ paraformaldehyde in a phosphate buffer $(\mathrm{PB} ; 0.1 \mathrm{M}$, 
$\mathrm{pH} 7.4 \pm 0.1$ ). Brains were removed and placed in the same fixative for post-fixation at $4^{\circ} \mathrm{C}$ for 6 hours. For cryoprotection, the samples were kept with cold $30 \%$ sucrose in PBS at $4{ }^{\circ} \mathrm{C}$ until samples sank to the bottom. Coronal brain sections $(30 \mu \mathrm{m})$ were cut using a cryostat and then floated in PBS. Sections were blocked with 5\% normal donkey serum in $0.3 \%$ Triton X-100/PBS for 1 hour at room temperature. Free-floating sections were incubated overnight at $4{ }^{\circ} \mathrm{C}$ on a rocker with one of the following primary antibodies: 1:100 IDO1 rabbit polyclonal (Santa Cruz Biotechnology Inc.); 1:1,000 NeuN mouse monoclonal (Chemicon); 1:1,000 GFAP mouse monoclonal (BD Biosciences - Pharmingen); 1:1,000 Iba-1 mouse monoclonal (Abcam). Sections were then washed with PBS 3 times and incubated with 1:300 FITC- or cyanine 3-conjugated secondary antibody (Jackson ImmunoResearch Laboratories Inc.) for 1 hour at room temperature. Blue fluorescent DAPI (1:300; Invitrogen) was used to stain the nucleus of fixed, cultured Neuro2a cells for 15 minutes.

Colocalization was examined by adding a second primary antibody following the same procedure as described above. Controls were performed by either omitting primary antibody or using antigen absorption (Supplementary Figure 3B). Images were examined with a fluorescence microscope (Olympus) and captured with a digital camera. The images were analyzed using Adobe Photoshop (version 7).

\section{Western blot analysis}

Rats were sacrificed under pentobarbital anesthesia. The hippocampus was separated from the brain and immediately stored on dry ice and kept at $-80^{\circ} \mathrm{C}$ until use. Samples were homogenized in a lysis buffer with SDS containing a cocktail of proteinase inhibitors (Roche). Samples were separated on an SDS-PAGE gel (4\%-15\% gradient gel; Bio-Rad) and transferred to PVDF membranes (Millipore). Membranes were blocked with $5 \%$ nonfat dried milk for 1 hour at room temperature and incubated overnight at $4{ }^{\circ} \mathrm{C}$ on a rocker with one of following primary antibodies: 1:200 IDO1 (Santa Cruz Biotechnology Inc.; rabbit polyclonal antibody), 1:200 IDO1 (Novus Biologicals; rabbit polyclonal antibody), 1:500 JAK2 (Santa Cruz Biotechnology Inc.; rabbit polyclonal antibody), 1:1,000 STAT3 (Millipore; rabbit polyclonal antibody), 1:5,000 p-STAT3 (Abcam; rabbit monoclonal antibody). After membranes were washed with PBS 4 times, membranes were incubated with 1:8,000 HRP-conjugated secondary antibody (Amersham Biosciences, GE Healthcare) for 1 hour at room temperature on a rocker. The membranes were washed with PBS 4 times, and blots were visualized in enhanced chemiluminescent (ECL) solution (Pierce) for 5 minutes and exposed to hyperfilms (Kodak) for 15 minutes. Blots were again incubated in a stripping buffer (Pierce) for 15 minutes at room temperature on a rocker and reprobed with 1:12,000 anti- $\beta$-actin antibody (Abcam Inc.) as a loading control. Western blots were made in triplicate. Band density was measured and normalized against a loading control band.

\section{ELISA}

Under pentobarbital anesthesia, whole blood was collected from the left ventricle of the heart and placed in a serum collection tube (BD Vacutainer). Blood clots were formed after 30-60 minutes at room temperature, and the tube was centrifuged for 10 minutes at 9,659.52 g. An ELISA Quantikine kit (R\&D Systems) and IDO ELISA kit (TSZ ELISA) were used according to the manufacturers' instructions. Optical density of samples was read at wavelengths of 450 and $570 \mathrm{~nm}$ using a microplate reader (Synergy HT, BioTEK). A standard curve, provided by the manufacturer, was generated for each set of samples assayed, and the concentration of each sample was obtained according to the standard curve.

\section{Real-time RT-PCR}

Total RNA was isolated from hippocampal samples using TRIzol reagent (Invitrogen). Four micrograms of total RNA was used to synthesize the first-strand cDNA using a SuperScript III kit (Invitrogen). Four microliters of cDNA was put into a $20-\mu l$ vessel using $1 \mu \mathrm{l}$ of $\times 20$ TaqMan Gene Expression Assay. TaqMan Gene Expression Assays (Applied Biosystems) containing primers and a TaqMan probe were used to quantify each gene of interest. The genes examined were Il6 (Rn01410330 m1, M26744.1) and Ido1 (Rn00576778 m1, AF312699.1) for rats and Il6 (Mm00446190 m1, $\mathrm{X} 54542.1)$ and $I d o 1$ (Mm00492586 m1, M69109.1) for mice. The reaction was performed in duplicate with the following conditions: denaturing at $95^{\circ} \mathrm{C}$ for 30 seconds, annealing at $60^{\circ} \mathrm{C}$ for 2 minutes, extension at $68^{\circ} \mathrm{C}$ for 2 minutes for 40 cycles using a 7300 Real-Time PCR System (Applied Biosystems). The RNA expression of GAPDH was measured as control.

\section{HPLC}

Hippocampal tryptophan, kynurenine, and serotonin content was determined by HPLC $(78,79)$. All samples were taken between 2 and 5 p.m. Kynurenine was measured by a UV detector (Shimadzu, SPD-10Avp; 360-nm wavelength). Tryptophan and serotonin were measured by a fluorescence detector (Shimadzu, RF-10Axl; 286-nm excitation and 366-nm emission wavelengths). Each day, external calibration was made by using freshly prepared control samples of the same concentrations (100 nmol/1 tryptophan, $100 \mu \mathrm{mol} / 1$ kynurenine, and $100 \mathrm{nmol} / 1$ serotonin).

\section{Statistics}

Repeated measures 2-way ANOVA was used and followed by the Tukey test to detect interactions between test time points and groups. Correlations between nociceptive and depressive behavior were determined using Pearson correlation coefficient analysis. HPLC, Western blot, and ELISA data were analyzed using 1-way ANOVA followed by a Tukey or Dunn test. Statistical analyses were performed using SigmaStat (Systat, version 11), with a significance level of $P<0.05$.

\section{Study approval}

Informed consent was obtained from study participants. Research protocols were approved by the Massachusetts General Hospital Institutional Animal Care and Use Committee. Experiments were conducted with the experimenter being unaware of the group assignment.

\section{Acknowledgments}

This study was supported by NIH grants R01DE18214, R01DE18538, and P20DA26002 (to J. Mao).

Received for publication November 11, 2011, and accepted in revised form May 16, 2012.

Address correspondence to: Jianren Mao, MGH Center for Translational Pain Research, Department of Anesthesia, Critical Care and Pain Medicine, Massachusetts General Hospital, Harvard Medical School, Boston, Massachusetts 02114, USA. Phone: 617.726.2338; Fax: 617.724.2719; E-mail: jmao@partners.org.
1. Sullivan MJ, Reesor K, Mikail S, Fisher R. The treatment of depression in chronic low back pain: review and recommendations. Pain. 1992;50(1):5-13.

2. Von KM, Simon G. The relationship between pain and depression. Br J Psychiatry Suppl. 1996; (30):101-108.

3. Mao J, Gold MS, Backonja MM. Combination drug therapy for chronic pain: a call for more clinical studies. J Pain. 2011;12(2):157-166.

4. Ganong AH, Cotman CW. Kynurenic acid and quinolinic acid act at $\mathrm{N}$-methyl-D-aspartate receptors in the rat hippocampus. J Pharmacol Exp Ther. 
1986;236(1):293-299

5. Dantzer R, O'Connor JC, Freund GG, Johnson RW, Kelley KW. From inflammation to sickness and depression: when the immune system subjugates the brain. Nat Rev Neurosi. 2008;9(1):46-56.

6. Popov A, et al. Indoleamine 2,3-dioxygenaseexpressing dendritic cells form suppurative granulomas following Listeria monocytogenes infection. J Clin Invest. 2006;116(12):3160-3170.

7. Heyes MP, et al. Quinolinic acid and kynurenine pathway metabolism in inflammatory and non-inflammatory neurological disease. Brain. 1992;115(pt 5):1249-1273.

8. Heyes MP, Lackner A. Increased cerebrospinal fluid quinolinic acid, kynurenic acid, and L-kynurenine in acute septicemia. J Neurochem. 1990;55(1):338-341.

9. Yoshida R, Urade Y, Tokuda M, Hayaishi O. Induction of indoleamine 2,3-dioxygenase in mouse lung during virus infection. Proc Natl Acad SciUS A. 1979;76(8):4084-4086

10. O'Connor JC, et al. Interferon-gamma and tumor necrosis factor-alpha mediate the upregulation of indoleamine 2,3-dioxygenase and the induction of depressive-like behavior in mice in response to bacillus Calmette-Guerin. J Neurosci. 2009;29(13):4200-4209.

11. De Jongh RF, Vissers KC, Meert TF, Booij LH, De Deyne CS, Heylen RJ. The role of interleukin-6 in nociception and pain. Anesth Analg. 2003; 96(4):1096-1103.

12. Maes M, Verkerk R, Bonaccorso S, Ombelet W, Bosmans E, Scharpe S. Depressive and anxiety symptoms in the early puerperium are related to increased degradation of tryptophan into kynurenine, a phenomenon which is related to immune activation. Life Sci. 2002;71(16):1837-1848.

13. Butler SH, Godefroy F, Besson JM, Weil-Fugazza J. A limited arthritic model for chronic pain studies in the rat. Pain. 1992;48(1):73-81.

14. Porsolt RD, Le PM, Jalfre M. Depression: a new animal model sensitive to antidepressant treatments. Nature. 1977;266(5604):730-732.

15. Hall CS. Emotional behavior in rat. J Comp Physiol Psychol. 1934;18(3):385-403.

16. Pare WP. Open field, learned helplessness, conditioned defensive burying, and forced-swim tests in WKY rats. Physiol Behav. 1994;55(3):433-439.

17. Guillemin GJ, Smythe G, Takikawa O, Brew BJ. Expression of indoleamine 2,3-dioxygenase and production of quinolinic acid by human microglia, astrocytes, and neurons. Glia. 2005;49(1):15-23.

18. Roy EJ, Takikawa O, Kranz DM, Brown AR, Thomas DL. Neuronal localization of indoleamine 2,3-dioxygenase in mice. Neurosci Lett. 2005;387(2):95-99.

19. O'Connor JC, et al. Lipopolysaccharide-induced depressive-like behavior is mediated by indoleamine 2,3-dioxygenase activation in mice. Mol Psychiatry. 2009;14(5):511-522.

20. Hama AT, Sagen J. Cannabinoid receptor-mediated antinociception with acetaminophen drug combinations in rats with neuropathic spinal cord injury pain. Neuropharmacology. 2010;58(4-5):758-766.

21. Mitchell D, Gelgor L, Weber J, Kamerman PR. Antihypernociceptive synergy between ibuprofen, paracetamol and codeine in rats. Eur J Pharmacol. 2010;642(1-3):86-92

22. Wang S, Lim G, Mao J, Sung B, Mao J. Regulation of the trigeminal NR1 subunit expression induced by inflammation of the temporomandibular joint region in rats. Pain. 2009;141(1-2):97-103.

23. Heinrich PC, Behrmann I, Muller-Newen G, Schaper F, Graeve L. Interleukin-6-type cytokine signalling through the gp130/Jak/STAT pathway. Biochem J. 1998;334(pt 2):297-314.

24. Legendre F, Dudhia J, Pujol JP, Bogdanowicz P. JAK/STAT but not ERK1/ERK2 pathway mediates interleukin (IL)-6/soluble IL-6R down-regulation of Type II collagen, aggrecan core, and link protein transcription in articular chondrocytes. Association with a down-regulation of SOX9 expression. J Biol Chem. 2003;278(5):2903-2912.

25. Wallenius K, Wallenius V, Sunter D, Dickson SL, Jansson JO. Intracerebroventricular interleukin-6 treatment decreases body fat in rats. Biochem Biophys Res Commun. 2002;293(1):560-565.

26. Dominguez E, Rivat C, Pommier B, Mauborgne A, Pohl M. JAK/STAT3 pathway is activated in spinal cord microglia after peripheral nerve injury and contributes to neuropathic pain development in rat. J Neurochem. 2008;107(1):50-60.

27. Dworkin RH, Gitlin MJ. Clinical aspects of depression in chronic pain patients. Clin J Pain. 1991;7(2):79-94.

28. Delgado PL. Common pathways of depression and pain. J Clin Psychiatry. 2004;65(suppl 12):16-19.

29. Dohrenwend BP, Raphael KG, Marbach JJ, Gallagher RM. Why is depression comorbid with chronic myofascial face pain? A family study test of alternative hypotheses. Pain. 1999;83(2):183-192.

30. Blackburn-Munro G, Blackburn-Munro RE. Chronic pain, chronic stress and depression: coincidence or consequence? J Neuroendocrinol. 2001;13(12):1009-1023.

31. Schatzberg AF. The relationship of chronic pain and depression. J Clin Psychiatry. 2004;65(suppl 12):3-4.

32. Jasmin L, Rabkin SD, Granato A, Boudah A, Ohara PT. Analgesia and hyperalgesia from GABA-mediated modulation of the cerebral cortex. Nature. 2003;424(6946):316-320.

33. Andre J, Zeau B, Pohl M, Cesselin F, Benoliel JJ, Becker C. Involvement of cholecystokininergic systems in anxiety-induced hyperalgesia in male rats: behavioral and biochemical studies. J Neurosci. 2005;25(35):7896-7904.

34. Gameiro GH, da Silva AA, Nouer DF, Ferraz de Arruda Veiga MC. How may stressful experiences contribute to the development of temporomandibular disorders? Clin Oral Investig. 2006;10(4):261-268.

35. Tsigos C, Chrousos GP. Hypothalamic-pituitary-adrenal axis, neuroendocrine factors and stress. J Psychosom Res. 2002;53(4):865-871.

36. Max MB, et al. Amitriptyline relieves diabetic neuropathy pain in patients with normal or depressed mood. Neurology. 1987;37(4):589-596.

37. Sharav Y, Singer E, Schmidt E, Dionne RA, Dubner $R$. The analgesic effect of amitriptyline on chronic facial pain. Pain. 1987;31(2):199-209.

38. Atkinson $\mathrm{JH}$, et al. A placebo-controlled randomized clinical trial of nortriptyline for chronic low back pain. Pain. 1998;76(3):287-296.

39. Zhang L, Zhang Y, Zhao ZQ. Anterior cingulate cortex contributes to the descending facilitatory modulation of pain via dorsal reticular nucleus. Eur J Neurosci. 2005;22(5):1141-1148.

40. Vogt BA. Pain and emotion interactions in subregions of the cingulate gyrus. Nat Rev Neurosci. 2005;6(7):533-544.

41. Derbyshire SW, Jones AK, Gyulai F, Clark S, Townsend D, Firestone LL. Pain processing during three levels of noxious stimulation produces differential patterns of central activity. Pain. 1997; 73(3):431-445.

42. Ploghaus A, et al. Exacerbation of pain by anxiety is associated with activity in a hippocampal network. J Neurosci. 2001;21(24):9896-9903.

43. Miura H, Ozaki N, Sawada M, Isobe K, Ohta T, Nagatsu T. A link between stress and depression: shifts in the balance between the kynurenine and serotonin pathways of tryptophan metabolism and the etiology and pathophysiology of depression. Stress. 2008;11(3):198-209.

44. Myint AM, Kim YK. Cytokine-serotonin interaction through IDO: a neurodegeneration hypothesis of depression. Med Hypotheses. 2003;61(5-6):519-525.

45. Mico JA, Gibert-Rahola J, Casas J, Rojas O, Serrano MI, Serrano JS. Implication of beta 1- and beta 2-adrenergic receptors in the antinociceptive effect of tricyclic antidepressants. Eur Neuropsychopharmacol. 1997;7(2):139-145.

46. Sawynok J, Esser MJ, Reid AR. Antidepressants as analgesics: an overview of central and peripheral mechanisms of action. $J$ Psychiatry Neurosci. 2001;26(1):21-29.

47. Stone TW, Perkins MN. Quinolinic acid: a potent endogenous excitant at amino acid receptors in CNS. Eur J Pharmacol. 1981;72(4):411-412.

48. Kwidzinski E, et al. Indolamine 2,3-dioxygenase is expressed in the CNS and down-regulates autoimmune inflammation. FASEB J. 2005; 19(10):1347-1349.

49. Yan Y, et al. IDO upregulates regulatory $\mathrm{T}$ cells via tryptophan catabolite and suppresses encephalitogenic $\mathrm{T}$ cell responses in experimental autoimmune encephalomyelitis. J Immunol. 2010; 185(10):5953-5961.

50. Hori T, Oka T, Hosoi M, Aou S. Pain modulatory actions of cytokines and prostaglandin E2 in the brain. Ann N Y Acad Sci. 1998;840:269-281.

51. Lenczowski MJ, et al. Central administration of rat IL-6 induces HPA activation and fever but not sickness behavior in rats. Am J Physiol. 1999; 276(3 pt 2):R652-R658.

52. Huang Y, Henry CJ, Dantzer R, Johnson RW, Godbout JP. Exaggerated sickness behavior and brain proinflammatory cytokine expression in aged mice in response to intracerebroventricular lipopolysaccharide. Neurobiol Aging. 2008;29(11):1744-1753.

53. Zeng Q, et al. Exacerbated mechanical allodynia in rats with depression-like behavior. Brain Res. 2008;1200:27-38

54. Norman GJ, Karelina K, Zhang N, Walton JC, Morris JS, Devries AC. Stress and IL-1beta contribute to the development of depressive-like behavior following peripheral nerve injury. Mol Psychiatry. 2010;15(4):404-414.

55. Yalcin I, et al. A time-dependent history of mood disorders in a murine model of neuropathic pain. Biol Psychiatry. 2011;70(10):946-953.

56. Pallotta MT, et al. Indoleamine 2,3-dioxygenase is a signaling protein in long-term tolerance by dendritic cells. Nat Immunol. 2011;12(9):870-878.

57. Kiefer R, Kieseier BC, Stoll G, Hartung HP. The role of macrophages in immune-mediated damage to the peripheral nervous system. Prog Neurobiol. 2001;64(2):109-127.

58. Smith PC, Hobisch A, Lin DL, Culig Z, Keller ET. Interleukin- 6 and prostate cancer progression. Cytokine Growth Factor Rev. 2001;12(1):33-40.

59. Connor TJ, Leonard BE. Depression, stress and immunological activation: the role of cytokines in depressive disorders. Life Sci. 1998;62(7):583-606.

60. Thomas AJ, Davis S, Morris C, Jackson E, Harrison $\mathrm{R}$, O'Brien JT. Increase in interleukin-1beta in latelife depression. Am J Psychiatry. 2005;162(1):175-177.

61. Hou DY, et al. Inhibition of indoleamine 2,3-dioxygenase in dendritic cells by stereoisomers of 1-methyl-tryptophan correlates with antitumor responses. Cancer Res. 2007;67(2):792-801.

62. Okamoto A, et al. Indoleamine 2,3-dioxygenase serves as a marker of poor prognosis in gene expression profiles of serous ovarian cancer cells. Clin Cancer Res. 2005;11(16):6030-6039.

63. Metz R, Duhadaway JB, Kamasani U, Laury-Kleintop L, Muller AJ, Prendergast GC. Novel tryptophan catabolic enzyme IDO2 is the preferred biochemical target of the antitumor indoleamine 2,3-dioxygenase inhibitory compound D-1-methyl-tryptophan. Cancer Res. 2007;67(15):7082-7087.

64. Paxinos G, Watson C. The Rat Brain in Stereotaxic Coordinates. New York, New York, USA: Academic Press; 1998.

65. Krsiak M. Timid singly-housed mice: their value in prediction of psychotropic activity of drugs. $\mathrm{BrJ}$ Pharmacol. 1975;55(1):141-150. 
66. Strekalova T, Spanagel R, Bartsch D, Henn FA, Gass P. Stress-induced anhedonia in mice is associated with deficits in forced swimming and exploration. Neuropsychopharmacology. 2004;29(11):2007-2017.

67. Rygula R, Abumaria N, Flugge G, Fuchs E, Ruther E, Havemann-Reinecke U. Anhedonia and motivational deficits in rats: impact of chronic social stress. Behav Brain Res. 2005;162(1):127-134

68. Hargreaves K, Dubner R, Brown F, Flores C, Joris $\mathrm{J}$. A new and sensitive method for measuring thermal nociception in cutaneous hyperalgesia. Pain. 1988;32(1):77-88.

69. Tal M, Bennett GJ. Extra-territorial pain in rats with a peripheral mononeuropathy: mechano-hyperalgesia and mechano-allodynia in the territory of an uninjured nerve. Pain. 1994;57(3):375-382.

70. Steru L, Chermat R, Thierry B, Simon P. The tail suspension test: a new method for screening antidepressants in mice. Psychopharmacology (Berl).
1985;85(3):367-370.

71. Denenberg VH. Open-field beavior in the rat: what does it mean? Ann N Y Acad Sci. 1969;159(3):852-859.

72. Willner P, Towell A, Sampson D, Sophokleous S, Muscat R. Reduction of sucrose preference by chronic unpredictable mild stress, and its restoration by a tricyclic antidepressant. Psychopharmacology (Berl). 1987;93(3):358-364.

73. Sigwalt AR, et al. Molecular aspects involved in swimming exercise training reducing anhedonia in a rat model of depression. Neuroscience. 2011; 192:661-674.

74. Zorina Y, Iyengar R, Bromberg KD. Cannabinoid 1 receptor and interleukin- 6 receptor together induce integration of protein kinase and transcription factor signaling to trigger neurite outgrowth. J Biol Chem. 2010;285(2):1358-1370.

75. Stoppini L, Buchs PA, Muller D. A simple method for organotypic cultures of nervous tissue. J Neuro- sci Methods. 1991;37(2):173-182.

76. Fu X, Zunich SM, O’Connor JC, Kavelaars A, Dantzer R, Kelley KW. Central administration of lipopolysaccharide induces depressive-like behavior in vivo and activates brain indoleamine 2,3 dioxygenase in murine organotypic hippocampal slice cultures. J Neuroinflammation. 2010;7:43.

77. Hakkoum D, Stoppini L, Muller D. Interleukin-6 promotes sprouting and functional recovery in lesioned organotypic hippocampal slice cultures. J Neurochem. 2007;100(3):747-757.

78. Widner B, Werner ER, Schennach $\mathrm{H}$, Wachter $\mathrm{H}$, Fuchs D. Simultaneous measurement of serum tryptophan and kynurenine by HPLC. Clin Chem. 1997:43(12):2424-2426.

79. Laich A, Neurauter G, Widner B, Fuchs D. More rapid method for simultaneous measurement of tryptophan and kynurenine by HPLC. Clin Chem. 2002;48(3):579-581. 